\title{
A Non-Stationary 3-D Wideband Twin-Cluster Model for 5G Massive MIMO Channels
}

\author{
Shangbin Wu, Cheng-Xiang Wang, Senior Member, IEEE, el-Hadi M. Aggoune, Senior Member, IEEE, \\ Mohammed M. Alwakeel, Senior Member, IEEE, and Yejun He, Senior Member, IEEE
}

\begin{abstract}
This paper proposes a novel theoretical non-stationary three dimensional (3-D) wideband twin-cluster channel model for massive multiple-input multiple-output (MIMO) communication systems with carrier frequencies on the order of gigahertz $(\mathrm{GHz})$. As the dimension of antenna arrays cannot be ignored for massive MIMO, near field effects instead of far field effects are considered in the proposed model. These include the spherical wavefront assumption and a birth-death process to model non-stationary properties of clusters such as cluster appearance and disappearance on both the array and time axes. Their impacts on massive MIMO channels are investigated via statistical properties including correlation functions, condition numbers, and angular power spectra. Additionally, the impact of elevation angles on correlation functions is discussed. A corresponding simulation model for the theoretical model is also proposed. Finally, numerical analysis shows that the proposed channel models are able to serve as a design framework for massive MIMO channel modeling.
\end{abstract}

Index Terms-Massive MIMO, 3-D twin-cluster channel model, spherical wavefront, non-stationarity, birth-death process.

\section{INTRODUCTION}

$\mathbf{M}$ ULTIPLE-INPUT multiple-output (MIMO) technologies are of great importance in modern wireless communication systems as they are able to substantially increase spectral efficiency [1]-[3]. Recently, massive MIMO systems [4]-[6], which are equipped with tens or even hundreds of

Manuscript received December 1, 2013; revised April 25, 2014; accepted May 25, 2014. Date of publication June 2, 2014; date of current version July 14, 2014. This work was supported in part by the SNCS Research Center, by the University of Tabuk, by the Ministry of Education of China through the Opening Project of the Key Laboratory of Cognitive Radio and Information Processing at Guilin University of Electronic Technology under Grant 2013KF01, by the Ministry of Science and Technology of China through the 863 Project in 5G Wireless Networking under Grant SS2014AA012101, by the Fundamental Research Program of Shenzhen City under Grants JCYJ20120817163755061 and JC201005250067A, and by the National Natural Science Foundation of China under Grant 61372077.

$\mathrm{S}$. Wu is with the Institute of Sensors, Signals and Systems, School of Engineering and Physical Sciences, Heriot-Watt University, Edinburgh EH14 4AS, U.K. He is also an Affiliate Associate Researcher of the SNCS Research Center, University of Tabuk, Saudi Arabia (e-mail: sw271@hw.ac.uk).

C.-X. Wang is with the School of Information Science and Engineering, Shandong University, Jinan 250100, China, and the Institute of Sensors, Signals and Systems, School of Engineering and Physical Sciences, HeriotWatt University, Edinburgh EH14 4AS, U.K. He is also an Affiliate Associate Researcher of the SNCS Research Center, University of Tabuk, Saudi Arabia (e-mail: cheng-xiang.wang@hw.ac.uk).

e.-H. M. Aggoune and M. M. Alwakeel are with the Sensor Networks and Cellular Systems Research Center, University of Tabuk, 71491 Tabuk, Saudi Arabia (e-mail: haggoune.sncs@ut.edu.sa; alwakeel@ut.edu.sa).

Y. He is with the College of Information Engineering, Shenzhen University, Shenzhen 518160, China (e-mail: heyejun@ieee.org).

Color versions of one or more of the figures in this paper are available online at http://ieeexplore.ieee.org.

Digital Object Identifier 10.1109/JSAC.2014.2328131 antennas, emerge as an enhanced MIMO technique to meet the increasing traffic demand of the fifth generation $(5 \mathrm{G})$ wireless communication networks [7], [8]. It was stated in [4]-[6] that massive MIMO systems have a number of additional benefits as compared with conventional MIMO systems which only have a small number of antenna elements. First, energy efficiency can be significantly increased by massive MIMO systems as they concentrate power on a sharp direction. Second, system throughput can be boosted by utilizing multi-user MIMO (MU-MIMO). Interference between users is averaged out by introducing a massive number of antennas according to the large number theorem. Third, implementation cost can be reduced by including simplification of medium-access control layer and deploying low-cost antenna elements due to great MU-MIMO and beamforming gain. Lastly, massive MIMO systems are more robust than conventional MIMO systems as they offer excessive degrees of freedom. As a result, massive MIMO is expected as an essential candidate technology for $5 \mathrm{G}$ wireless communication networks.

For the sake of MIMO system design and performance evaluation, it is indispensable to develop accurate and efficient small-scale fading MIMO channel models. For conventional MIMO systems, there are many small-scale fading channel models reported in the literature. Regular shape geometrybased stochastic models (GBSMs) such as one-ring, two-ring, and ellipse models can be found in [9]-[16], where the authors assumed that scatterers are distributed on regular shapes. Thus, channel impulse responses of these channel models were purely determined by the geometrical relationships between the scatterer and receiver (transmitter). Standardized GBSMs such as the spatial channel model (SCM) [17], the WINNER II model [18], and the international mobile telecommunicationsadvanced (IMT-Advanced) model [19] focus on the geometry of the first and the last bounces of scatterers. In addition, correlation-based stochastic models (CBSMs) [20]-[23], such as the Kronecker model and Weichselberger model, are usually utilized to study the performance of MIMO systems due to their low complexity.

However, the aforementioned conventional MIMO channel models are not suitable to be directly applied to modeling massive MIMO channels. Measurements on massive MIMO channels in [24] and [25] indicated that there are two characteristics making massive MIMO channels different from conventional MIMO channels. First, since the number of antennas is huge in massive MIMO systems, the far field assumption in conventional MIMO channels may no longer be appropriate. The distance between the receiver and transmitter (or a cluster) may not be beyond the Rayleigh distance defined by $2 \mathcal{L}^{2} / \lambda$ 
[26], where $\mathcal{L}$ and $\lambda$ are the dimension of the antenna array and carrier wavelength, respectively. For uniform linear arrays (ULAs) with a fixed antenna separation $\Delta$ (e.g., $\Delta=\lambda / 2$ ), the dimension $\mathcal{L}=(\mathcal{M}-1) \Delta$ is linearly increasing with the number of antenna elements $\mathcal{M}$. Therefore, the wavefront should be assumed as spherical instead of plane when the number of antennas is large. Although the impact of spherical wavefront on short-range or constant distance communications was studied in [27] and [28], its impact on massive MIMO channels has not yet been reported in the literature. Second, non-stationary properties can be observed on large antenna arrays [24], i.e., appearance and disappearance of clusters can occur on the array axis. This leads to the fact that each antenna element on the array may observe different sets of clusters, which is not characterized in conventional MIMO channels. As a result, the wide-sense stationary (WSS) assumption on antenna arrays does not necessarily hold for massive MIMO channels. The authors in [29]-[32] modeled cluster evolution on the time axis with birth-death processes or Markov processes. However, to the best of our knowledge, non-stationary properties of clusters on the array axis have not been studied for massive MIMO channels in the literature.

Additionally, it was reported in [33] that scatterers would disperse in elevation (or the vertical plane) and the impact of elevation angles needs to be addressed in realistic channel models. Therefore, three dimensional (3-D) channel models should be developed for massive MIMO systems. Extensive conventional 3-D MIMO models can be found in the literature such as the twin-cluster MIMO model [34], the COST 2100 model [35], [36], 3-D extension of the WINNER model [37], 3-D double-directional radio model [38], and 3-D MIMO vehicleto-vehicle channel model [39]. In this paper, we will extend the twin-cluster MIMO model in [34], where a cluster is divided into two representations of itself (one at the transmitter and the other at the receiver), by incorporating the spherical wavefront assumption, cluster evolution on the time and array axes, and 3-D cluster properties to capture massive MIMO channel characteristics.

The contributions of this paper are summarized as follows:

1) This paper first proposes a theoretical non-stationary 3-D wideband twin-cluster channel model for massive MIMO systems with carrier frequencies in the order of gigahertz $(\mathrm{GHz})$, i.e., they are not applicable to millimeter wave communication systems. To the best of the authors' knowledge, this is the first 3-D model for massive MIMO channels. An infinite number of scatterers are assumed in the proposed theoretical model (or reference model), which cannot be implemented in hardware or for simulation purposes. Therefore, we also propose a corresponding 3-D simulation model with finite numbers of scatterers. Various statistical properties, such as the spatial-temporal correlation function, Doppler power spectral density (PSD), and condition number, are studied for both the theoretical and simulation models. Numerical results have demonstrated that the statistical properties of the simulation model can fit those of the theoretical model very well with reasonable complexity. Also, the impact

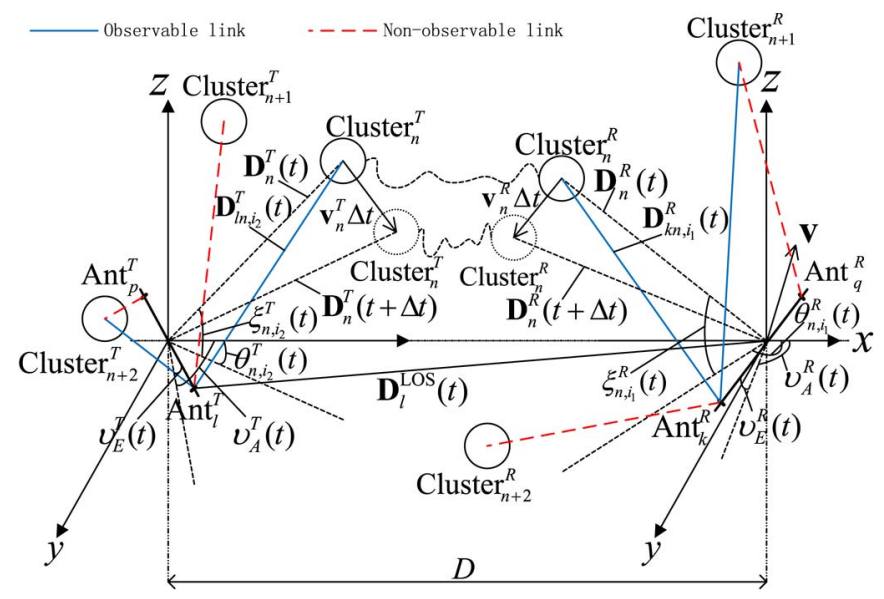

Fig. 1. A 3-D wideband twin-cluster massive MIMO channel model.

of cluster elevation angles on correlation properties of the proposed massive MIMO channel models is investigated.

2) In the proposed theoretical and simulation massive MIMO channel models, near field effects caused by the increasing antenna elements are considered, including the spherical wavefront assumption and the variation of Doppler frequencies over the antenna array.

3) Also, appearance and disappearance of clusters (i.e., cluster evolution) on both the array and time axes are jointly modeled by birth-death processes, which make the proposed massive MIMO channel models essentially non-stationary.

The rest of this paper is organized as follows. Section II gives a general description of the proposed theoretical non-stationary 3-D wideband twin-cluster channel model for massive MIMO systems. Statistical properties of the proposed theoretical model are studied in Section III. Section IV presents the corresponding simulation model for the theoretical model. Numerical results are presented in Section $\mathrm{v}$ and conclusions are finally drawn in Section VI.

\section{A Theoretical Non-Stationary 3-D Wideband Twin-Cluster Massive MiMo Channel Model}

Let us consider a wideband massive MIMO system with multiple twin clusters in a 3-D space to describe different taps of the channel, as illustrated in Fig. 1. For a twin-cluster channel model with $N_{\text {total }}$ clusters, each cluster, say $\operatorname{Cluster}_{n}(n=$ $\left.1, \ldots, N_{\text {total }}\right)$, is made of a representation Cluster $_{n}^{T}$ at the transmitter side denoting the first bounce and a representation Cluster $_{n}^{R}$ at the receiver side denoting the last bounce. The propagation environment between these two representations is abstracted as a virtual link [34].

Let us assume that the transmitter and receiver are equipped with ULAs with $M_{T}$ and $M_{R}$ antenna elements, respectively. The distances between antenna elements are $\delta_{T}$ at the transmitter and $\delta_{R}$ at the receiver. Let the transmitter be the origin of the 3-D space, the distance vector between the transmitter and receiver is $\mathbf{D}=(D, 0,0)$. Furthermore, both azimuth and elevation angles in the 3-D space are considered for clusters, antenna arrays, movement direction of clusters, and movement direction of antenna arrays as listed in Table I. It should be noticed that the far field conditions $\left(D>\left(2 M_{T}^{2} \delta_{T}^{2} / \lambda\right), D>\right.$ 
TABLE I

DeFinitions OF Key GeOMETRY PARAMETERS

\begin{tabular}{|c|c|}
\hline$v_{E}^{R}, v_{E}^{T}$ & elevation angles of the receive and transmit antenna arrays \\
\hline$v_{A}^{R}, v_{A}^{T}$ & azimuth angles of the receive and transmit antenna arrays \\
\hline$\xi_{n, i_{1}}^{R}, \theta_{n, i_{1}}^{R}$ & elevation and azimuth angles of the $i_{1}$-th ray of the $n$-th cluster at the receiver side \\
\hline$\xi_{n, i_{2}}^{T}, \theta_{n, i_{2}}^{T}$ & elevation and azimuth angles of the $i_{2}$-th ray of the $n$-th cluster at the transmitter side \\
\hline $\mathbf{D}_{n}^{R}(t), \mathbf{D}_{n}^{T}(t)$ & distance vectors between the $n$-th cluster and the receiver (transmitter) \\
\hline $\mathbf{D}_{n, i_{1}}^{R}(t)$ & distance vector between the $n$-th cluster and the receive antenna array via the $i_{1}$-th ray \\
\hline $\mathbf{D}_{k n, i_{1}}^{R}(t)$ & distance vector between the $n$-th cluster and the $k$-th receive antenna via the $i_{1}$-th ray \\
\hline $\mathbf{D}_{n, i_{2}}^{T}(t)$ & distance vector between the $n$-th cluster and the transmit antenna array via the $i_{2}$-th ray \\
\hline $\mathbf{D}_{l n, i_{2}}^{T}(t)$ & distance vector between the $n$-th cluster and the $l$-th transmit antenna via the $i_{2}$-th ray \\
\hline $\mathbf{D}_{k l}^{L O S}(t)$ & distance vector between $k$-th receive antenna and the $l$-th transmit antenna \\
\hline $\mathbf{v}$ & velocity vector of the receive antenna array \\
\hline $\mathbf{v}_{n}^{R}, \mathbf{v}_{n}^{T}$ & velocity vectors of the the $n$-th cluster at the receiver and transmitter side \\
\hline
\end{tabular}

$\left.\left(2 M_{R}^{2} \delta_{R}^{2} / \lambda\right)\right)$ for conventional MIMO channels are not assumed in the proposed model. Thus, the wavefront of each wireless link is assumed to be spherical resulting in the fact that the AoAs and AoDs on the antenna arrays are no longer equal for each antenna element and the phase of each antenna element is determined by geometrical relationships.

Another important characteristic of massive MIMO channel models is the appearance and disappearance of clusters on the antenna array as reported in [24]. Contrary to conventional MIMO channel models assuming that a cluster is always observable to all the antennas on an antenna array, a cluster may only be observable to a subset of antennas on an antenna array for massive MIMO. To put it another way, each antenna has its own set of observable clusters. Examples are shown in Fig. 1. Cluster $_{n+1}$ is observable to the $k$ th but not observable to the $q$ th $\left(1 \leqslant k, q \leqslant M_{R}\right)$ receive antenna. Similarly, Cluster $_{n+2}$ is observable to the $l$ th but not observable to the $p$ th $(1 \leqslant l, p \leqslant$ $\left.M_{T}\right)$ transmit antenna. On the other hand, Cluster ${ }_{n}$ is observable to both the $l$ th transmit antenna and the $k$ th receive antenna. Denote $C_{l}^{T}(t)\left(C_{k}^{R}(t)\right)$ as the cluster set in which clusters are observable to the $l$ th transmit antenna (the $k$ th receive antenna) at time instant $t$. Let $N_{\text {total }}$ be the total number of clusters that are observable to at least one transmit antenna and one receive antenna. The value of $N_{\text {total }}$ can be calculated as

$$
N_{\text {total }}=\operatorname{card}\left(\bigcup_{l=1}^{M_{T}} \bigcup_{k=1}^{M_{R}}\left(C_{l}^{T}(t) \bigcap C_{k}^{R}(t)\right)\right)
$$

where the operator card $(\cdot)$ denotes the cardinality of a set. Then, a cluster is observable to the $l$ th transmit antenna and the $k$ th receive antenna if and only if this cluster is in the set $\left\{C_{l}^{T}(t) \bigcap C_{k}^{R}(t)\right\}$. Sets $C_{l}^{T}(t)$ and $C_{k}^{R}(t)$ are generated based on the cluster evolution (birth-death process) on both the time and array axes as described in Section II-B.

\section{A. Channel Impulse Response}

Next, let us denote the maximum Doppler frequency as $f_{\max }$, the line-of-sight (LOS) Rician factor as $K$, and the initial phase of the signal at the transmitter as $\varphi_{0}$. Additionally, let us assume that the power of the $n$th cluster is $P_{n}$ and there are, respectively $S_{1}$ and $S_{2}$ rays within the representation at the receiver side and the representation at the transmitter side. Based on geometrical parameters in Table I, as $S_{1}, S_{2} \rightarrow \infty$, the theoretical model of the wideband massive MIMO channel matrix can be represented as an $M_{R} \times M_{T}$ complex matrix $\mathbf{H}(t, \tau)=\left[h_{k l}(t, \tau)\right]_{M_{R} \times M_{T}}$ where $k=1,2, \ldots, M_{R}$ and $l=$ $1,2, \ldots, M_{T}$. The multipath complex gains between the $l$ th transmit antenna and the $k$ th receive antenna at time $t$ and delay $\tau, h_{k l}(t, \tau)$, can be presented as

$$
h_{k l}(t, \tau)=\sum_{n=1}^{N_{\text {total }}} h_{k l, n}(t) \delta\left(\tau-\tau_{n}(t)\right)
$$

- if $\operatorname{Cluster}_{n} \in\left\{C_{l}^{T}(t) \cap C_{k}^{R}(t)\right\}$,

$$
\begin{gathered}
h_{k l, n}(t)=\underbrace{\delta(n-1) \sqrt{\frac{K}{K+1}} e^{j\left(2 \pi f_{k l}^{\mathrm{LOS}} t+\varphi_{k l}^{\mathrm{LOS}}\right)}}_{\text {NLOS }} \\
+\underbrace{\sqrt{\frac{P_{n}}{K+1}} \lim _{S_{1}, S_{2} \rightarrow \infty} \sum_{i_{1}=1}^{S_{1}} \sum_{i_{2}=1}^{S_{2}} \frac{e^{j\left(2 \pi f_{k n, i_{1}} t+\varphi_{k l, n, i_{1} i_{2}}\right)}}{\sqrt{S_{1} S_{2}}}}_{\text {LOS }} \\
- \text { if } \operatorname{Cluster}_{n} \notin\left\{C_{l}^{T}(t) \cap C_{k}^{R}(t)\right\}, \\
h_{k l, n}(t)=0 .
\end{gathered}
$$

The calculation of complex gains can be divided into non-lineof-sight (NLOS) components and LOS component.

1) For NLOS Components: The $k$ th receive antenna vector $\mathbf{A}_{k}^{R}(t)$ and the vector between the $n$th cluster and the receive antenna array via the $i_{1}$-th ray $\mathbf{D}_{n, i_{1}}^{R}(t)$ can be presented as

$$
\begin{aligned}
\mathbf{A}_{k}^{R}(t) & =\frac{M_{R}-2 k+1}{2} \delta_{R}\left[\begin{array}{c}
\cos v_{E}^{R}(t) \cos v_{A}^{R}(t) \\
\cos v_{E}^{R}(t) \sin v_{A}^{R}(t) \\
\sin v_{E}^{R}(t)
\end{array}\right]^{\mathrm{T}}+\mathbf{D} \\
\mathbf{D}_{n, i_{1}}^{R}(t) & =D_{n}^{R}(t)\left[\begin{array}{c}
\cos \xi_{n, i_{1}}^{R}(t) \cos \theta_{n, i_{1}}^{R}(t) \\
\cos \xi_{n, i_{1}}^{R}(t) \sin \theta_{n, i_{1}}^{R}(t) \\
\sin \xi_{n, i_{1}}^{R}(t)
\end{array}\right]^{\mathrm{T}}+\mathbf{D} .
\end{aligned}
$$

Similarly, the $l$ th transmit antenna vector $\mathbf{A}_{l}^{T}$ and the vector between the $n$th cluster and the transmit antenna array via the $i_{2}$-th ray $\mathbf{D}_{n, i_{2}}^{T}(t)$ can be given as

$$
\begin{gathered}
\mathbf{A}_{l}^{T}=\frac{M_{T}-2 l+1}{2} \delta_{T}\left[\begin{array}{c}
\cos v_{E}^{T} \cos v_{A}^{T} \\
\cos v_{E}^{T} \sin v_{A}^{T} \\
\sin v_{E}^{T}
\end{array}\right]^{\mathrm{T}} \\
\mathbf{D}_{n, i_{2}}^{T}(t)=D_{n}^{T}(t)\left[\begin{array}{c}
\cos \xi_{n, i_{2}}^{T}(t) \cos \theta_{n, i_{2}}^{T}(t) \\
\cos \xi_{n, i_{2}}^{T}(t) \sin \theta_{n, i_{2}}^{T}(t) \\
\sin \xi_{n, i_{2}}^{T}(t)
\end{array}\right]^{\mathrm{T}} .
\end{gathered}
$$




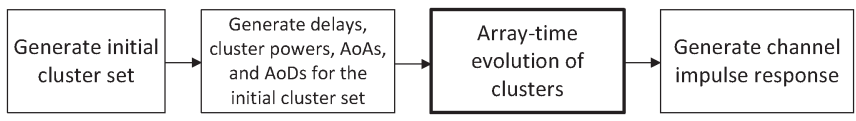

Fig. 2. Algorithm flowchart of the generation of the channel impulse response.

Then, vectors $\mathbf{D}_{k n, i_{1}}^{R}(t)$ and $\mathbf{D}_{l n, i_{2}}^{T}(t)$ can be computed as

$$
\begin{aligned}
\mathbf{D}_{k n, i_{1}}^{R}(t) & =\mathbf{D}_{n, i_{1}}^{R}(t)-\mathbf{A}_{k}^{R}(t) \\
\mathbf{D}_{l n, i_{2}}^{T}(t) & =\mathbf{D}_{n, i_{2}}^{T}(t)-\mathbf{A}_{l}^{T} .
\end{aligned}
$$

Next, the delay of the $n$th cluster of the twin-cluster model is assumed to be the sum of two components. The first component is calculated according to the geometrical relationships between the antenna arrays and cluster locations. The second component abstracts the delay of the virtual link between the twin clusters. Then, the delay of the $n$th cluster $\tau_{n}(t)$ can be computed as

$$
\tau_{n}(t)=\frac{\left\|\mathbf{D}_{n}^{T}(t)\right\|+\left\|\mathbf{D}_{n}^{R}(t)\right\|}{c}+\tilde{\tau}_{n}(t)
$$

where the abstracted delay of the virtual link $\tilde{\tau}_{n}(t)$ is randomly drawn according to the uniform distribution $U\left(D / c, \tau_{\max }\right)$, and $\tau_{\max }$ is the maximum delay ( $\tau_{\max }=1845 \mathrm{~ns}$ for NLOS [18]). The operator $\|\cdot\|$ denotes the Euclidean norm, and $c$ is the speed of light. Then, the phase between the $k$ th receive antenna and the $l$ th transmit antenna via the $i_{1}$-th ray at the receiver, the $i_{2}$-th ray at the transmitter, and the $n$th cluster, $\varphi_{k l, n, i_{1} i_{2}}(t)$, is derived as

$\varphi_{k l, n, i_{1} i_{2}}(t)=\varphi_{0}+\frac{2 \pi}{\lambda}\left[\left\|\mathbf{D}_{k n, i_{1}}^{R}(t)\right\|+\left\|\mathbf{D}_{l n, i_{2}}^{T}(t)\right\|+c \tilde{\tau}_{n}(t)\right]$.

Accordingly, the Doppler frequency of the $k$ th receive antenna via the $i_{1}$-th ray of the $n$th cluster $f_{k n, i_{1}}(t)$ is presented as

$$
f_{k n, i_{1}}(t)=\frac{f_{\max }\left\langle\mathbf{D}_{k n, i_{1}}^{R}(t), \mathbf{v}\right\rangle}{\left\|\mathbf{D}_{k n, i_{1}}^{R}(t)\right\|\|\mathbf{v}\|}
$$

where $\langle\cdot, \cdot\rangle$ represents the inner product.

2) For LOS Component: In the same way, the Doppler frequency $f_{k l}^{\mathrm{LOS}}(t)$ and phase $\varphi_{k l}^{\mathrm{LOS}}(t)$ of the LOS components can also be calculated as

$$
\begin{aligned}
\mathbf{D}_{k l}^{\mathrm{LOS}}(t) & =\mathbf{A}_{k}^{R}(t)-\mathbf{A}_{l}^{T} \\
f_{k l}^{\mathrm{LOS}}(t) & =\frac{f_{\max }\left\langle\mathbf{D}_{k l}^{\mathrm{LOS}}(t), \mathbf{v}\right\rangle}{\left\|\mathbf{D}_{k l}^{\mathrm{LOS}}(t)\right\|\|\mathbf{v}\|} \\
\varphi_{k l}^{\mathrm{LOS}}(t) & =\varphi_{0}+\frac{2 \pi}{\lambda}\left\|\mathbf{D}_{k l}^{\mathrm{LOS}}(t)\right\| .
\end{aligned}
$$

The generation procedure of the channel impulse response consists of the generation of the initial cluster set, generation of parameters (delays, cluster powers, AoAs, and AoDs) for the initial cluster set, array-time evolution of clusters, and the generation of channel impulse response, as presented in Fig. 2. This algorithm is a generalized version of the WINNER channel model [18] by adding an extra block of array-time evolution of clusters to capture massive MIMO channel characteristics. The block of array-time evolution of clusters will be discussed in the next section.

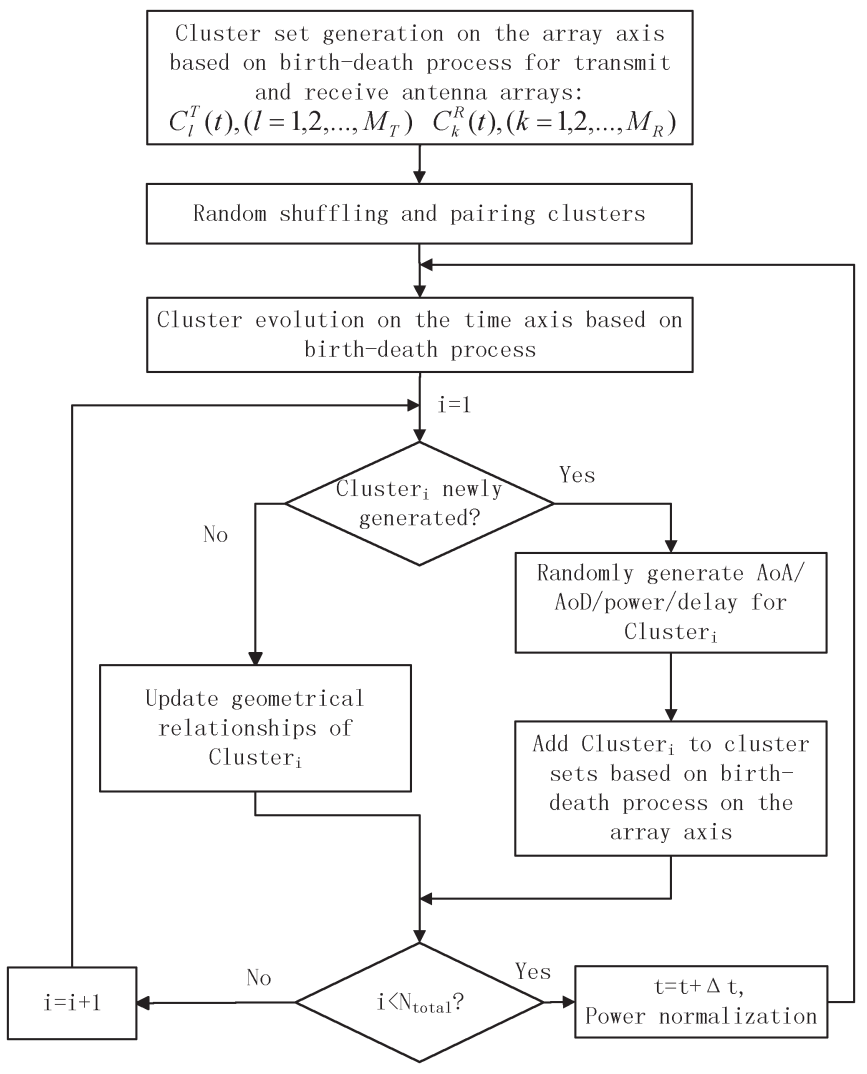

Fig. 3. Algorithm flowchart of array-time evolution of the proposed 3-D twincluster model.

\section{B. Non-Stationary Properties}

The non-stationary process of the proposed massive MIMO channel model is based on the array-time evolution of clusters which can be characterized by two parts. The first part is the generation of cluster sets $C_{l}^{T}(t)$ and $C_{k}^{R}(t)$ for each antenna based on birth-death process on both the time and array axes. This aims at modeling not only the phenomena of cluster appearance and disappearance on antenna arrays of massive MIMO, but also non-stationary behaviors of clusters on the time axis. The generation procedure is achieved by extending the concept of birth-death process on the time axis in previous literature [29], [30] to the array axis as well. The outcome of the first part determines the cluster set of each antenna. The second part is the updates of geometrical relationships with respect to the movements of the receiver and clusters. The outcome of the second part determines all parameters for each cluster. The algorithm flowchart describing the array-time cluster evolution is depicted in Fig. 3.

Part 1: To describe the algorithm of the array-time cluster evolution, let us first denote $\lambda_{G}$ (per meter) and $\lambda_{R}$ (per meter) as the cluster generation rate and recombination rate. Assume the initial number of clusters $N$ and the initial cluster sets of the first transmit and receive antennas $C_{1}^{T}=\left\{c_{x}^{T}: x=\right.$ $1,2, \ldots, N\}$ and $C_{1}^{R}=\left\{c_{x}^{R}: x=1,2, \ldots, N\right\}$ at the initial time instant $t$ are given, where $c_{x}^{T}$ and $c_{x}^{R}$ are two representations of Cluster ${ }_{x}$. Then, these clusters in cluster sets $C_{1}^{T}$ and $C_{1}^{R}$ evolve according to birth-death process on the array axis to recursively generate the cluster sets of the rest of antennas at 
TABLE II

Distributions of Key Geometry Parameters

\begin{tabular}{|c|c|c|c|}
\hline Parameters & Distributions & Mean & Standard deviation \\
\hline $\bar{\xi}_{n}^{T}$ & wrapped Gaussian & $0.78 \mathrm{rad}$ & $0.11 \mathrm{rad}$ \\
\hline $\bar{\theta}_{n}^{T}$ & wrapped Gaussian & $1.05 \mathrm{rad}$ & $0.53 \mathrm{rad}$ \\
\hline $\bar{\xi}_{n}^{R}$ & wrapped Gaussian & $0.78 \mathrm{rad}$ & $0.18 \mathrm{rad}$ \\
\hline $\bar{\theta}_{n}^{R}$ & wrapped Gaussian & $0.78 \mathrm{rad}$ & $0.91 \mathrm{rad}$ \\
\hline $\mathbf{D}_{n}^{R}(t)$ & exponential & $25 \mathrm{~m}$ & $0.07 \mathrm{~m}$ \\
\hline $\mathbf{D}_{n}^{T}(t)$ & exponential & $30 \mathrm{~m}$ & $0.05 \mathrm{~m}$ \\
\hline$\tau_{n}$ & exponential & $930 \mathrm{~ns}$ & $930 \mathrm{~ns}$ \\
\hline
\end{tabular}

the transmitter and receiver at the initial time instant $t$, which is expressed as

$$
\begin{array}{ll}
C_{l-1}^{T}(t) \stackrel{\mathrm{E}}{\rightarrow} C_{l}^{T}(t) & \left(l=2,3, \ldots, M_{T}\right) \\
C_{k-1}^{R}(t) \stackrel{\mathrm{E}}{\rightarrow} C_{k}^{R}(t) & \left(k=2,3, \ldots, M_{R}\right)
\end{array}
$$

where the operator $\stackrel{\mathrm{E}}{\rightarrow}$ denotes cluster evolution on either the array or time axis. The survival probabilities of the clusters inside the cluster set the on array axis at the transmitter $P_{\text {survival }}^{T}$ and the receiver $P_{\text {survival }}^{R}$ can be modeled as exponential functions [40]

$$
\begin{gathered}
P_{\text {survival }}^{T}=e^{-\lambda_{R} \frac{\delta_{T}}{D_{c}^{a}}} \\
P_{\text {survival }}^{R}=e^{-\lambda_{R} \frac{\delta_{R}}{D_{c}^{a}}}
\end{gathered}
$$

where $D_{c}^{a}$ is the scenario-dependent correlation factor on the array axis. According to the birth-death process, the average number of newly generated clusters $N_{\text {new }}^{T}$ and $N_{\text {new }}^{R}$ on the array axis based on the birth-death process can be computed as [40]

$$
\begin{aligned}
& \mathrm{E}\left[N_{\text {new }}^{T}\right]=\frac{\lambda_{G}}{\lambda_{R}}\left(1-e^{-\frac{\delta_{T}}{D_{c}^{a}}}\right) \\
& \mathrm{E}\left[N_{\text {new }}^{R}\right]=\frac{\lambda_{G}}{\lambda_{R}}\left(1-e^{-\frac{\delta_{R}}{D_{c}^{c}}}\right)
\end{aligned}
$$

where $\mathrm{E}[\cdot]$ designates the expectation. After this process on the array axis, a number of initial clusters may not survive for certain antennas. Meanwhile, new clusters may appear on the array. Each cluster evolves gradually on the antenna array. It can be observed from (19)-(22) that, if two antenna elements are more separated, the probability that they share the same set of clusters is smaller. To imitate the complex propagation environment, cluster indices in set $\bigcup_{l=1}^{M_{T}} C_{l}^{T}$ and set $\bigcup_{k=1}^{M_{R}} C_{k}^{R}$ are randomly shuffled and paired to determine to which transmit and receive antennas each cluster is observable.

Then, the cluster indices are reassigned from 1 to $N_{\text {total }}$. Moreover, parameters of the initial clusters such as mean AoAs, mean AoDs, delays, and distances are randomly drawn according to distributions listed in Table II. The means and standard deviations for $\bar{\xi}_{n}^{T}, \bar{\theta}_{n}^{T}, \bar{\xi}_{n}^{R}, \bar{\theta}_{n}^{R}$, and $\tau_{n}$ in Table II are generated according to [18]. Also, the power of each cluster is calculated and normalized as in [18].
At the next time instant $t+\Delta t$, the time-axis evolution of clusters is operated as

$$
\begin{aligned}
& C_{l}^{T}(t) \stackrel{\mathrm{E}}{\rightarrow} C_{l}^{T}(t+\Delta t) \\
& C_{k}^{R}(t) \stackrel{\mathrm{E}}{\rightarrow} C_{k}^{R}(t+\Delta t) .
\end{aligned}
$$

To perform the evolution process of cluster on the time axis as (23) and (24) show, define the time-dependent channel fluctuation in the time axis at $t+\Delta t$ as $q(t+\Delta t)$. The channel fluctuation measures how much the scattering environment varies within a short period of time. The variation of scattering environment is due to the movements of the receiver and the clusters. Thus, the channel fluctuation is defined by [29]

$$
q(t+\Delta t)=q_{r}(t+\Delta t)+q_{c}(t+\Delta t)
$$

where $q_{r}(t+\Delta t)$ is the channel fluctuation caused by the movement of receiver defined by $q_{r}(t+\Delta t)=\|\mathbf{v}\| \Delta t$ and $q_{c}(t+\Delta t)$ is the channel fluctuation caused by the movement of clusters defined by $q_{c}(t+\Delta t)=P_{F}\left(\left\|\mathbf{v}_{n}^{T}\right\|+\left\|\mathbf{v}_{n}^{R}\right\|\right) \Delta t\left(P_{F}\right.$ is the percentage of moving clusters). Given the scenariodependent space correlation factor $D_{c}^{s}$, each cluster survives with probability $P_{\text {survival }}$ on the time axis which can be calculated as [29]

$$
P_{\text {survival }}(q(t+\Delta t))=e^{-\frac{\lambda_{R} q(t+\Delta t)}{D_{c}^{s}}} .
$$

The mean number of newly generated clusters at time instant $t+\Delta t$ on the time axis $\mathrm{E}\left[N_{\text {new }}(t+\Delta t)\right]$ is computed according to the birth-death process [40]

$$
\mathrm{E}\left[N_{\text {new }}(t+\Delta t)\right]=\frac{\lambda_{G}}{\lambda_{R}}\left(1-e^{-\frac{\lambda_{R} q(t+\Delta t)}{D_{c}^{s}}}\right) .
$$

After the time evolution process as (23)-(27) show, all clusters can be categorized as survived clusters or newly generated clusters. The next issue is to decide the set of transmit and receive antennas that are observable to each newly generated cluster. This is determined by the birth-death process on the array axis, which can be summarized into 4 steps:

Step 1: Randomly generate initial indices $\tilde{l}\left(1 \leqslant \tilde{l} \leqslant M_{T}\right)$ and $\tilde{k}\left(1 \leqslant \tilde{k} \leqslant M_{R}\right)$ for the transmit and receive antenna arrays. Then, let the newly generated cluster be observable to the $\tilde{l}$-th transmit antenna and the $\tilde{k}$-th receive antenna.

Step 2: Evolve the cluster on the transmit antenna array based on birth-death process from the $(\tilde{l}-1)$-th to the 1 -st and from $(\tilde{l}+1)$-th to the $M_{T}$-th antennas.

Step 3: Evolve the cluster on the receive antenna array based on birth-death process from the $(\tilde{k}-1)$-th to the 1 -st and from $(\tilde{k}+1)$-th to the $M_{R}$-th antennas.

Step 4: Add the cluster to cluster sets whose corresponding antennas can observe the cluster.

Part 2: The remaining issue is the updates of geometry relationships of clusters from $t$ to $t+\Delta t$. The updates of geometry relationships are different for survived clusters and newly generated clusters. Thus, they are described separately. 
1) Survived Clusters: Regarding survived clusters, their properties such as delays, Doppler frequencies, AoAs and AoDs should be recalculated based on the updates of geometrical relationships from $t$ to $t+\Delta t$. First, the distance vectors are updated due to movements of the receiver and clusters according to

$$
\begin{aligned}
\mathbf{D}_{n}^{R}(t+\Delta t) & =\mathbf{D}_{n}^{R}(t)+\mathbf{v}_{n}^{R} \Delta t \\
\mathbf{D}_{n}^{T}(t+\Delta t) & =\mathbf{D}_{n}^{T}(t)+\mathbf{v}_{n}^{T} \Delta t \\
\mathbf{D}_{k n, i_{1}}^{R}(t+\Delta t) & =\mathbf{D}_{n, i_{1}}^{R}(t)-\mathbf{A}_{k}^{R}(t)+\left(\mathbf{v}_{n}^{R}-\mathbf{v}\right) \Delta t \\
\mathbf{D}_{l n, i_{2}}^{T}(t+\Delta t) & =\mathbf{D}_{n, i_{2}}^{T}(t)+\mathbf{v}_{n}^{T} \Delta t-\mathbf{A}_{l}^{T} .
\end{aligned}
$$

Second, the delay of the $n$-th cluster at $t+\Delta t$ is expressed as the sum of the updated geometrical delay and the delay of the evolved virtual link,

$\tau_{n}(t+\Delta t)=\frac{\left\|\mathbf{D}_{n}^{T}(t+\Delta t)\right\|+\left\|\mathbf{D}_{n}^{R}(t+\Delta t)\right\|}{c}+\tilde{\tau}_{n}(t+\Delta t)$.

To describe the evolution of the virtual link, its delay $\tilde{\tau}_{n}(t+$ $\Delta t)$ is based on a first-order filtering algorithm as $\tilde{\tau}_{n}(t+\Delta t)=$ $e^{-(\Delta t / \varsigma)} \tilde{\tau}_{n}(t)+\left(1-e^{-(\Delta t / \varsigma)}\right) X$ where $X$ is randomly drawn according to the uniform distribution $U\left(D / c, \tau_{\max }\right), \varsigma$ is a parameter that depends on the coherence of a virtual link and scenarios. Third, the time-variant phase and Doppler frequency are accordingly computed as

$$
\begin{gathered}
\varphi_{k l, n, i_{1} i_{2}}(t+\Delta t)=\varphi_{0}+\frac{2 \pi}{\lambda} c \tilde{\tau}_{n}(t+\Delta t) \\
+\frac{2 \pi}{\lambda}\left[\left\|\mathbf{D}_{k n, i_{1}}^{R}(t+\Delta t)\right\|+\left\|\mathbf{D}_{l n, i_{2}}^{T}(t+\Delta t)\right\|\right] \\
f_{k n, i_{1}}(t+\Delta t)=\frac{f_{\max }\left\langle\mathbf{D}_{k n, i_{1}}^{R}(t+\Delta t), \mathbf{v}\right\rangle}{\left\|\mathbf{D}_{k n, i_{1}}^{R}(t+\Delta t)\right\|\|\mathbf{v}\|}
\end{gathered}
$$

Last, geometrical relationships of LOS components need to be refreshed as well

$$
\begin{aligned}
\mathbf{D}_{k l}^{\mathrm{LOS}}(t+\Delta t) & =\mathbf{A}_{k}^{R}(t)+\mathbf{v} \Delta t-\mathbf{A}_{l}^{T} \\
f_{k l}^{\mathrm{LOS}}(t+\Delta t) & =\frac{f_{\max }\left\langle\mathbf{D}_{k l}^{\mathrm{LOS}}(t+\Delta t), \mathbf{v}\right\rangle}{\left\|\mathbf{D}_{k l}^{\mathrm{LOS}}(t+\Delta t)\right\|\|\mathbf{v}\|} \\
\varphi_{k l}^{\mathrm{LOS}}(t+\Delta t) & =\varphi_{0}+\frac{2 \pi}{\lambda}\left\|\mathbf{D}_{k l}^{\mathrm{LOS}}(t+\Delta t)\right\| .
\end{aligned}
$$

2) Newly Generated Clusters: On the other hand, for newly generated clusters, their AoAs, AoDs, delays, and distances are initialized according to distributions in Table II. The power of each cluster is calculated and normalized as in [18]. Denote the set of all survived clusters as $C_{\text {Survived }}$ and the set of all newly generated clusters as $C_{\mathrm{New}}$ after the time-axis evolution. The average total power of survived and newly generated clusters should be normalized as

$$
\sum_{\text {Cluster }_{i} \in C_{\text {Survived }}} P_{i}+\sum_{\text {Cluster }_{j} \in C_{\text {New }}} P_{j}=1 .
$$

Thus far, the array-time evolution of clusters from $t$ to $t+\Delta t$ is finished. This evolution process can be operated recursively with respect to time.

\section{Statistical Properties of the Theoretical MASSIVE MiMO CHANNEL MODEL}

\section{A. Spatial-Temporal Correlation Function}

The spatial-temporal correlation function between the channel gains $h_{k l, n}(t)$ and $h_{k^{\prime} l^{\prime}, n}(t)$ is defined as [10]

$$
\rho_{k l, k^{\prime} l^{\prime}, n}\left(\delta_{T}, \delta_{R}, \Delta t ; t\right)=\mathrm{E}\left[\frac{h_{k l, n}^{*}(t) h_{k^{\prime} l^{\prime}, n}(t+\Delta t)}{\left|h_{k l, n}^{*}(t)\right|\left|h_{k^{\prime} l^{\prime}, n}(t+\Delta t)\right|}\right] .
$$

As the LOS component and NLOS components are independent, (39) can be rewritten as the sum of the spatial-temporal correlation functions of the LOS component and the NLOS components

$$
\begin{aligned}
& \rho_{k l, k^{\prime} l^{\prime}, n}\left(\delta_{T}, \delta_{R}, \Delta t ; t\right) \\
& \quad=\rho_{k l, k^{\prime} l^{\prime}, n}^{\mathrm{LOS}}\left(\delta_{T}, \delta_{R}, \Delta t ; t\right)+\rho_{k l, k^{\prime} l^{\prime}, n}^{\mathrm{NLOS}}\left(\delta_{T}, \delta_{R}, \Delta t ; t\right)
\end{aligned}
$$

where

$$
\begin{aligned}
& \rho_{k l, k^{\prime} l^{\prime}, n}^{\mathrm{LOS}}\left(\delta_{T}, \delta_{R}, \Delta t ; t\right)=\frac{K \delta(n-1)}{K+1} \\
& \times e^{j\left[2 \pi f_{k^{\prime} l^{\prime}}^{\mathrm{LOS}}(t+\Delta t)(t+\Delta t)-2 \pi f_{k l}^{\mathrm{LOS}}(t) t+\varphi_{k^{\prime} l^{\prime}}^{\mathrm{LOS}}(t+\Delta t)-\varphi_{k l}^{\mathrm{LOS}}(t)\right]} \\
& \rho_{k l, k^{\prime} l^{\prime}, n}^{\mathrm{LLOS}}\left(\delta_{T}, \delta_{R}, \Delta t ; t\right)=\frac{1}{K \delta(n-1)+1} \\
& \quad \times \mathrm{E}\left[\lim _{S_{1}, S_{2} \rightarrow \infty} \frac{1}{\sqrt{S_{1} S_{2}}} \sum_{i_{1}=1}^{S_{1}} \sum_{i_{2}=1}^{S_{2}} e^{j \Phi_{0}}\right]
\end{aligned}
$$

with

$$
\begin{aligned}
\Phi_{0}=2 \pi f_{k^{\prime} n, i_{1}}( & t+\Delta t)(t+\Delta t)-2 \pi f_{k n, i_{1}}(t) t \\
& +\varphi_{k^{\prime} l^{\prime}, n, i_{1} i_{2}}(t+\Delta t)-\varphi_{k l, n, i_{1} i_{2}}(t) .
\end{aligned}
$$

\section{B. Spatial Cross-Correlation Function}

By setting $\Delta t=0$, the spatial-temporal correlation function reduces to the spatial cross-correlation (CCF) function $\rho_{k l, k^{\prime} l^{\prime}, n}\left(\delta_{T}, \delta_{R} ; t\right)$.

$$
\begin{aligned}
\rho_{k l, k^{\prime} l^{\prime}, n}\left(\delta_{T}, \delta_{R} ; t\right) & =\mathrm{E}\left[\frac{h_{k l, n}^{*}(t) h_{k^{\prime} l^{\prime}, n}(t)}{\left|h_{k l, n}^{*}(t)\right|\left|h_{k^{\prime} l^{\prime}, n}(t)\right|}\right] \\
& =\rho_{k l, k^{\prime} l^{\prime}, n}^{\operatorname{LOS}}\left(\delta_{T}, \delta_{R} ; t\right)+\rho_{k l, k^{\prime} l^{\prime}, n}^{\mathrm{NLOS}}\left(\delta_{T}, \delta_{R} ; t\right)
\end{aligned}
$$

where

$$
\begin{aligned}
& \rho_{k l, k^{\prime} l^{\prime}, n}^{\mathrm{LOS}}\left(\delta_{T}, \delta_{R} ; t\right)=\frac{K \delta(n-1)}{K+1} \\
& \times e^{j\left[2 \pi f_{k^{\prime} l^{\prime}}^{\mathrm{LOS}}(t) t-2 \pi f_{k l}^{\mathrm{LOS}}(t) t+\varphi_{k^{\prime} l^{\prime}}^{\mathrm{LOS}}(t)-\varphi_{k l}^{\mathrm{LOS}}(t)\right]} .
\end{aligned}
$$

Regarding the correlation of the NLOS components, as a cluster has a probability of $e^{-\lambda_{R}\left(\left|l-l^{\prime}\right| \delta_{T}+\left|k-k^{\prime}\right| \delta_{R} / D_{c}^{a}\right)}$ to survive when 
evolving from $h_{k l, n}(t)$ to $h_{k^{\prime} l^{\prime}, n}(t)$, the spatial CCF of the

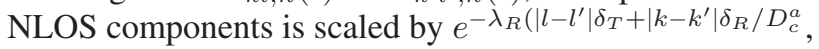

$$
\begin{aligned}
& \rho_{k l, k^{\prime} l^{\prime}, n}^{\mathrm{NLOS}}\left(\delta_{T}, \delta_{R} ; t\right)=\frac{1}{K \delta(n-1)+1} e^{-\lambda_{R} \frac{\left|l-l^{\prime}\right| \delta_{T}+\left|k-k^{\prime}\right| \delta_{R}}{D_{c}^{a}}} \\
& \times \int_{-\pi}^{\pi} \int_{-\pi}^{\pi} \int_{-\frac{\pi}{2}}^{\frac{\pi}{2}} \int_{-\frac{\pi}{2}}^{\frac{\pi}{2}} e^{j \Phi_{1}} p_{R}\left(\xi_{n}^{R}, \theta_{n}^{R}\right) p_{T}\left(\xi_{n}^{T}, \theta_{n}^{T}\right) d \xi_{n}^{R} d \xi_{n}^{T} d \theta_{n}^{R} d \theta_{n}^{T}
\end{aligned}
$$

with

$$
\Phi_{1}=2 \pi f_{k^{\prime} n}(t) t-2 \pi f_{k n}(t) t+\varphi_{k^{\prime} l^{\prime}, n}(t)-\varphi_{k l, n}(t) .
$$

As the spatial CCF $\rho_{k l, k^{\prime} l^{\prime}, n}\left(\delta_{T}, \delta_{R} ; t\right)$ depends on the values of $k, k^{\prime}, l, l^{\prime}$, and cannot be reduced to solely be with respect to $\left|k-k^{\prime}\right|$ and $\left|l-l^{\prime}\right|$, the WSS assumption on the array axis for massive MIMO is not valid.

\section{Temporal Autocorrelation Function}

On the other hand, by setting $l=l^{\prime}, k=k^{\prime}$, the temporal autocorrelation function (ACF) $\rho_{k l, n}(\Delta t ; t)$ is obtained.

$$
\begin{aligned}
\rho_{k l, n}(\Delta t ; t) & =\mathrm{E}\left[\frac{h_{k l, n}^{*}(t) h_{k l, n}(t+\Delta t)}{\left|h_{k l, n}^{*}(t)\right|\left|h_{k l, n}(t+\Delta t)\right|}\right] \\
& =\rho_{k l, n}^{\mathrm{LOS}}(\Delta t ; t)+\rho_{k l, n}^{\mathrm{NLOS}}(\Delta t ; t) .
\end{aligned}
$$

Since the LOS component is uncorrelated to NLOS components, their temporal ACFs, $\rho_{k l, n}^{\mathrm{LOS}}(\Delta t ; t)$ and $\rho_{k l, n}^{\mathrm{NLOS}}(\Delta t ; t)$, are calculated separately as

$$
\begin{aligned}
& \rho_{k l, n}^{\mathrm{LOS}}(\Delta t ; t)=\frac{K \delta(n-1)}{K+1} \\
& \quad \times e^{j\left[2 \pi f_{k l}^{\mathrm{LOS}}(t+\Delta t)(t+\Delta t)-2 \pi f_{k l}^{\mathrm{LOS}}(t) t+\varphi_{k l}^{\mathrm{LOS}}(t+\Delta t)-\varphi_{k l}^{\mathrm{LOS}}(t)\right] .}
\end{aligned}
$$

Regarding the correlation of the NLOS components, the survival probability of a cluster is $e^{-\lambda_{R}\left(\|\mathbf{v}\| \Delta t+P_{F}\left(\left\|\mathbf{v}_{n}^{T}\right\|+\left\|\mathbf{v}_{n}^{R}\right\|\right) \Delta t / D_{c}^{s}\right)}$ when evolving from $h_{k l, n}(t)$ to $h_{k l, n}(t+\Delta t)$, the temporal ACF of the NLOS components is scaled by $e^{-\lambda_{R}\left(\|\mathbf{v}\| \Delta t+P_{F}\left(\left\|\mathbf{v}_{n}^{T}\right\|+\left\|\mathbf{v}_{n}^{R}\right\|\right) \Delta t / D_{c}^{s}\right)}$,

$$
\begin{aligned}
& \rho_{k l, n}^{\mathrm{NLOS}}(\Delta t ; t)=\frac{1}{K \delta(n-1)+1} e^{-\lambda_{R} \frac{|\mathbf{v}| \Delta t+P_{F}\left(\left|\mathbf{v}_{n}^{T}\right|+\left|\mathbf{v}_{n}^{R}\right|\right) \Delta t}{D_{c}^{s}}} \\
& \times \int_{-\pi}^{\pi} \int_{-\pi}^{\pi} \int_{-\frac{\pi}{2}}^{\frac{\pi}{2}} \int_{-\frac{\pi}{2}}^{\frac{\pi}{2}} e^{j \Phi_{2}} p_{R}\left(\xi_{n}^{R}, \theta_{n}^{R}\right) p_{T}\left(\xi_{n}^{T}, \theta_{n}^{T}\right) d \xi_{n}^{R} d \xi_{n}^{T} d \theta_{n}^{R} d \theta_{n}^{T}
\end{aligned}
$$

with

$$
\begin{aligned}
\Phi_{2}=2 \pi f_{k n}(t+\Delta t)(t+\Delta t)-2 \pi f_{k n}(t) t & \\
& +\varphi_{k l, n}(t+\Delta t)-\varphi_{k l, n}(t) .
\end{aligned}
$$

As the temporal ACF $\rho_{k l, n}(\Delta t ; t)$ depends on the values of $t$, and cannot be reduced to solely be with respect to $\Delta t$, the WSS assumption on the time axis for the proposed massive MIMO channel model is not valid.

\section{Doppler Power Spectral Density}

The Doppler PSD $S_{n}(f ; t)$ with respect to the Doppler frequency $f$ is the Fourier transform of the temporal ACF, which can be presented as

$$
S_{n}(f ; t):=\int_{-\infty}^{\infty} \rho_{k l, n}(\Delta t ; t) e^{-j 2 \pi f \Delta t} d(\Delta t) .
$$

It should be also noticed that the Doppler PSD is time dependent.

\section{E. Doppler Frequency Standard Deviation on the Antenna Array}

As spherical wavefronts are assumed in the proposed channel model, different antennas on the same array will experience different Doppler shifts. Namely, Doppler frequencies may vary on the antenna array. To study the variations of Doppler frequency of the receiver on the array axis, the average Doppler frequency on the $k$ receive antenna, $\bar{f}_{k n}$, is calculated as

$$
\bar{f}_{k n}=\int_{-\pi}^{\pi} \int_{-\frac{\pi}{2}}^{\frac{\pi}{2}} f_{k n}\left(\xi_{n}^{R}, \theta_{n}^{R}\right) p\left(\xi_{n}^{R}, \theta_{n}^{R}\right) d \xi_{n}^{R} d \theta_{n}^{R} .
$$

Next, the average Doppler frequency on the array axis $\mu_{\bar{f}_{n}}$ is presented as $\mu_{\bar{f}_{n}}=\mathrm{E}\left[\bar{f}_{k n}\right]=\sum_{k=1}^{M_{R}} \bar{f}_{k n} / M_{R}$. Finally, the standard deviation of Doppler frequency $\sigma_{\bar{f}_{n}}$ on the array axis can be obtained as

$$
\sigma_{\bar{f}_{n}}=\sqrt{\frac{\sum_{k=1}^{M_{R}}\left(\bar{f}_{k n}-\mu_{\bar{f}_{n}}\right)^{2}}{M_{R}}} .
$$

For conventional MIMO channel models, the Doppler frequency on the whole antenna array is assumed to be the same which is equivalent to $\sigma_{\bar{f}_{n}}=0$. Conversely, for massive MIMO channel models, $\sigma_{\bar{f}_{n}}$ may not be 0 and a larger $\sigma_{\bar{f}_{n}}$ means that the Doppler frequency varies more significantly on the antenna array.

\section{F. Condition Number}

Condition number is used to measure the correlation of the channel matrix [4]. A larger condition number implies higher correlation. The condition number is defined by the quotient of maximum eigenvalue and the minimum eigenvalue of the channel matrix

$$
\gamma(\mathrm{dB})=20 \log _{10} \frac{\lambda_{\max }(\mathbf{H})}{\lambda_{\min }(\mathbf{H})}
$$

where the operators $\lambda_{\max }(\cdot)$ and $\lambda_{\min }(\cdot)$ represent the maximum eigenvalue and the minimum eigenvalue, respectively.

\section{A Non-Stationary 3-D Wideband Simulation MODEL FOR MASSIVE MIMO CHANNELS}

Previously, in the proposed channel theoretical model, the number of scatterers is assumed to be infinity $\left(S_{1}, S_{2} \rightarrow \infty\right)$ 
which is capable of providing accurate analytic channel characteristic results. However, with respect to a channel simulator, infinite scatterers are not practical as the complexity of implementation is enormous. Therefore, a compromise between accuracy and complexity should be addressed. The target of this section is to develop a channel simulator with a finite and proper scatterer number while capturing channel characteristics as accurate as possible. The corresponding simulation model of the proposed massive MIMO channel model is obtained by reducing (3) as

$$
\begin{aligned}
h_{k l, n}(t)= & \delta(n-1) \sqrt{\frac{K}{K+1}} e^{j\left(2 \pi f_{k l}^{\mathrm{LOS}} t+\varphi_{k l}^{\mathrm{LOS}}\right)} \\
& +\sqrt{\frac{P_{n}}{K+1}} \sum_{i_{1}=1}^{S_{1}} \sum_{i_{2}=1}^{S_{2}} \frac{e^{j\left(2 \pi f_{k n, i_{1}} t+\varphi_{k l, n, i_{1} i_{2}}\right)}}{\sqrt{S_{1} S_{2}}} .
\end{aligned}
$$

Let $\mathbf{x}$ be the vector of AoAs and AoDs defined by $\mathbf{x}=$ $\left(\theta^{R}, \xi^{R}, \theta^{T}, \xi^{T}\right)$. In the theoretical model, define $g(\mathbf{x})=$ $\left(h_{k l, n}^{*}(t) h_{k^{\prime} l^{\prime}, n}(t+\Delta t)\right) /\left(\left|h_{k l, n}^{*}(t)\right|\left|h_{k^{\prime} l^{\prime}, n}(t+\Delta t)\right|\right)$ with $\mathbf{x}$ distributed according to the cumulative distribution function (CDF) $F(\mathbf{x})$ of AoAs and AoDs. The spatial-temporal correlation function $\rho_{k l, k^{\prime} l^{\prime}, n}\left(\delta_{T}, \delta_{R}, \Delta t ; t\right)$ for the theoretical model in (39) is calculated as the expectation of $g(\mathbf{x})$,

$$
\rho_{k l, k^{\prime} l^{\prime}, n}\left(\delta_{T}, \delta_{R}, \Delta t ; t\right)=\mathrm{E}[g(\mathbf{x})]=\int g(\mathbf{x}) d F(\mathbf{x}) .
$$

On the other hand, a simulation model aims at approximating $\rho_{k l, k^{\prime} l^{\prime}, n}\left(\delta_{T}, \delta_{R}, \Delta t ; t\right)$ with $S_{1} S_{2}$ discrete vectors $\left\{\mathbf{x}_{i}\right\}_{i=1}^{S_{1} S_{2}}$, where each $\mathbf{x}_{i}$ follows the $\operatorname{CDF} F(\mathbf{x})$ and $\mathbf{x}_{i}=\left(\theta_{i}^{R}, \xi_{i}^{R}, \theta_{i}^{T}, \xi_{i}^{T}\right)$. Then, the approximated value $\hat{\rho}_{k l, k^{\prime} l^{\prime}, n}\left(\delta_{T}, \delta_{R}, \Delta t ; t\right)$ can be expressed as

$$
\hat{\rho}_{k l, k^{\prime} l^{\prime}, n}\left(\delta_{T}, \delta_{R}, \Delta t ; t\right)=\mathrm{E}[g(\mathbf{x})]=\frac{1}{S_{1} S_{2}} \sum_{i=1}^{S_{1} S_{2}} g\left(\mathbf{x}_{i}\right) .
$$

The remaining issue is to determine the vector sequence $\left\{\mathbf{x}_{i}\right\}_{i=1}^{S_{1} S_{2}}$ with reasonable computational complexity to approximate $\rho_{k l, k^{\prime} l^{\prime}, n}\left(\delta_{T}, \delta_{R}, \Delta t ; t\right)$ with $\hat{\rho}_{k l, k^{\prime} l^{\prime}, n}\left(\delta_{T}, \delta_{R}, \Delta t ; t\right)$. A number of algorithms to calculate $\left\{\mathbf{x}_{i}\right\}_{i=1}^{S_{1} S_{2}}$ such as the method of equal distances (MED), method of equal areas (MEA), extended method of exact Doppler spread (MEDS), and the Monte Carlo Method (MCM) have been introduced in [10]. Here, the MEA is applied to calculating the discrete vectors $\left\{\mathbf{x}_{i}\right\}_{i=1}^{S_{1} S_{2}}$ of the simulation model according to $\int_{\mathbf{x}_{i-1}}^{\mathbf{x}_{i}} d F(\mathbf{a})=1 / S_{1} S_{2}$ [10].

Since $\mathrm{x}$ is four dimensional in this model, the 'area' in MEA in this case is generalized as the probability measure of a set. As a result, $F(\mathbf{x})$ is divided into $S_{1} S_{2}$ sets with the same probability of $1 / S_{1} S_{2}$, then $\mathbf{x}_{i}$ can be computed as $\mathbf{x}_{i}=$ $F^{-1}\left(i / S_{1} S_{2}\right)$ where $F^{-1}(\cdot)$ is the inverse function of $F(\cdot)$.

\section{NumericAl RESUlts AND ANALysis}

The distributions of azimuth and elevation angles at the transmitter side and the receiver side are assumed to obey the two dimensional (2-D) von Mises distribution [41], where azimuth angles and elevation angles are assumed to be mutually independent. Therefore, the probability density function (PDF)

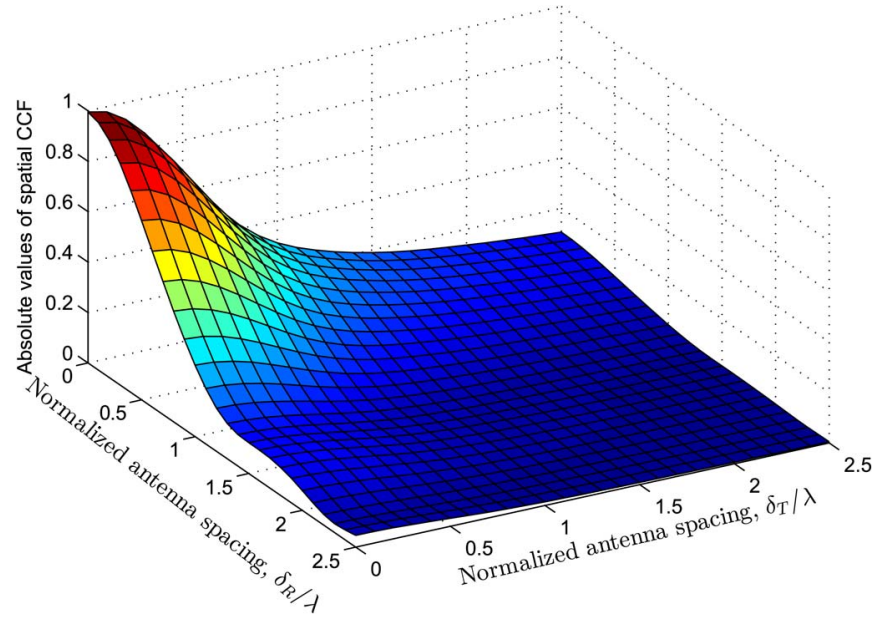

Fig. 4. Absolute values of the spatial CCF $\left|\rho_{11,22,1}\left(\delta_{T}, \delta_{R} ; t\right)\right|$ of the 3-D twin-cluster model. $\left(M_{R}=M_{T}=32, D=200 \mathrm{~m}, D_{c}^{a}=30 \mathrm{~m}, D_{c}^{s}=50 \mathrm{~m}\right.$, $v_{A}^{T}=\pi / 3, v_{E}^{T}=\pi / 6, v_{A}^{R}=\pi / 4, v_{E}^{R}=\pi / 4, \bar{\theta}_{1}^{T}=-1.03, \bar{\xi}_{1}^{T}=1.19, \bar{\theta}_{1}^{R}=$ $1.65, \bar{\xi}_{1}^{R}=0.16, t=4 \mathrm{~s}, \lambda=0.15 \mathrm{~m}, f_{\max }=33.33 \mathrm{~Hz}, \alpha_{v}=\pi / 6, \kappa=5$, NLOS).

of angles of the $n$th cluster $p_{Z}\left(\xi_{n}^{Z}, \theta_{n}^{Z}\right)$ with $Z=\{T, R\}$ can be expressed as

$$
p_{Z}\left(\xi_{n}^{Z}, \theta_{n}^{Z}\right)=\frac{\exp \left[\kappa\left(\cos \left(\xi_{n}^{Z}-\bar{\xi}_{n}^{Z}\right)+\cos \left(\theta_{n}^{Z}-\bar{\theta}_{n}^{Z}\right)\right)\right]}{\left[2 \pi I_{0}(\kappa)\right]^{2}}
$$

where $\bar{\xi}_{n}^{Z}$ and $\bar{\theta}_{n}^{Z}$ are the mean elevation and azimuth AoD/AoA, and $I_{0}(\cdot)$ is the zero-th order modified Bessel function. Moreover, $\kappa \geqslant 0$ controls the width of the distribution functions.

By setting $\Delta t$ to 0 , the absolute values of the spatial CCF $\left|\rho_{11,22,1}\left(\delta_{T}, \delta_{R} ; t\right)\right|$ of the three dimensional twin cluster model are illustrated in Fig. 4. A decreasing trend can be observed as the normalized antenna spacings increase at both the transmitter and receiver sides. The absolute values of spatial CCF drop smoothly when antenna spacing at the transmitter side enlarges. Meanwhile, fluctuations can be seen as antenna spacing at the receiver side increases. These fluctuations are caused by nonstationary properties due to the movements of the receiver.

Next, by setting $\delta_{T}$ and $\Delta t$ to 0 , the impact of cluster elevation angles at the receiver side on the absolute spatial correlation function $\left|\rho_{11,12,1}\left(0, \delta_{R} ; t\right)\right|$ of the receiver is depicted in Fig. 5. The increase in cluster elevation angles at the receiver side results in high receive antenna correlations. Besides, the spatial correlation function of the simulation model is compared with the theoretical model, showing that the simulation model is able to capture the channel spatial correlation characteristic at the cost of slightly less accuracy.

The absolute values of the temporal ACF in terms of cluster elevation angles at the receiver side are analyzed in Fig. 6. The figure shows that the temporal ACF decreases slower as the elevation angles become larger. The philosophy is that the Doppler frequency equals the product of $f_{\max }$, cosine of the azimuth angle, and cosine of the elevation angle. For fixed $f_{\max }$ and azimuth angle, the absolute Doppler frequency is decreasing as the elevation angle increases from 0 to $\pi / 2$. Consequently, when the elevation angle reaches $\pi / 2$, the absolute Doppler frequency is minimum which results in the slowest decrease of the temporal ACF. Meanwhile, the normalized 


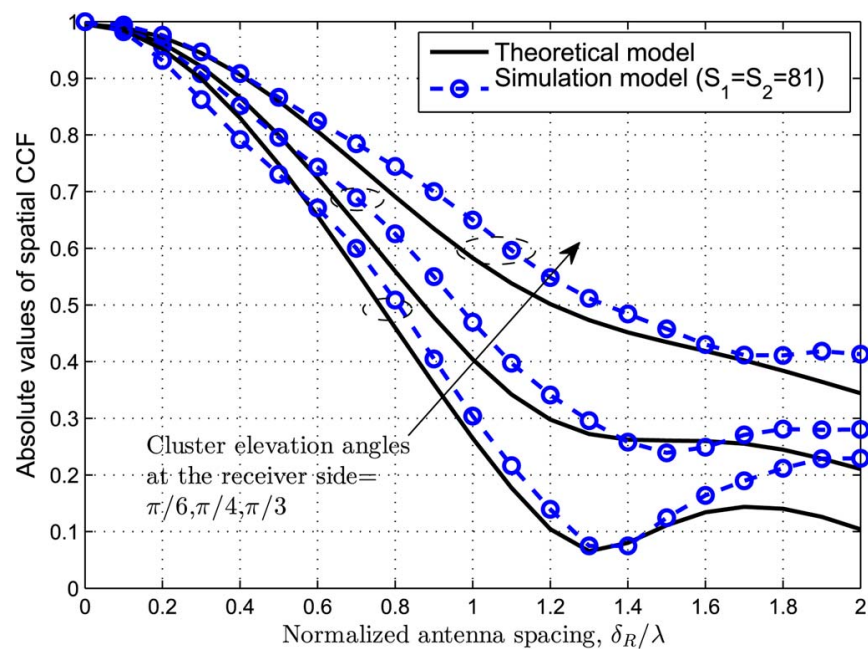

Fig. 5. Absolute values of the receiver spatial CCF $\left|\rho_{11,12,1}\left(0, \delta_{R} ; t\right)\right|$ in terms of cluster elevation angles at the receiver side. $\left(M_{R}=32, D=200 \mathrm{~m}\right.$, $D_{c}^{a}=30 \mathrm{~m}, D_{c}^{s}=50 \mathrm{~m}, \varsigma=1 \mathrm{~s}, v_{A}^{T}=\pi / 3, v_{E}^{T}=\pi / 6, v_{A}^{R}=\pi / 4, v_{E}^{R}=$ $\pi / 4, \bar{\theta}_{1}^{T}=-1.03, \bar{\xi}_{1}^{T}=1.19, \bar{\theta}_{1}^{R}=1.65, t=4 \mathrm{~s}, \lambda=0.15 \mathrm{~m}, f_{\max }=$ $33.33 \mathrm{~Hz}, \alpha_{v}=\pi / 6, \kappa=5$, NLOS).

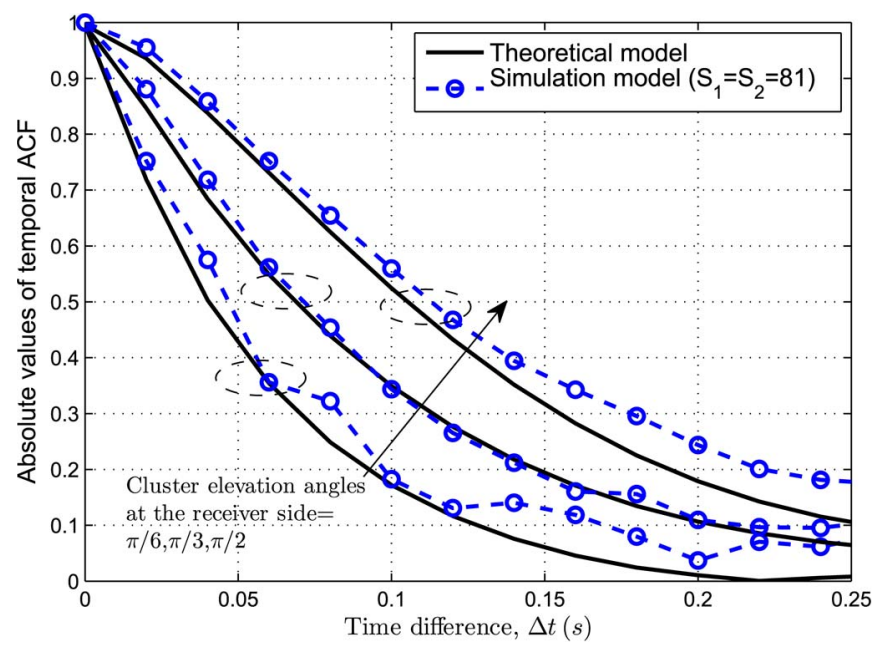

Fig. 6. Absolute values of the temporal ACF $\left|\rho_{11,1}(\Delta t ; t)\right|$ in terms of cluster elevation angle at the receiver side $\left(M_{R}=32, D=200 \mathrm{~m}, D_{c}^{a}=30 \mathrm{~m}\right.$, $D_{c}^{s}=50 \mathrm{~m}, \varsigma=1 \mathrm{~s}, v_{A}^{T}=\pi / 3, v_{E}^{T}=\pi / 6, v_{A}^{R}=\pi / 4, v_{E}^{R}=\pi / 4, \bar{\theta}_{1}^{T}=$ $-1.03, \bar{\xi}_{1}^{T}=1.19, \bar{\theta}_{1}^{R}=1.65, t=4 \mathrm{~s}, \lambda=0.15 \mathrm{~m},\left\|\mathbf{v}_{n}^{T}\right\|=\left\|\mathbf{v}_{n}^{R}\right\|=$ $0.25 \mathrm{~m} / \mathrm{s}, P_{F}=0.3, f_{\max }=33.33 \mathrm{~Hz}, \alpha_{v}=\pi / 6, \kappa=5$, NLOS).

Fourier transform of the temporal ACF, i.e., the PDF of Doppler frequency of the proposed model is illustrated in Fig. 7. The PDF of Doppler frequency of the conventional MIMO channel model is symmetrical with respect to 0 . However, this may not be necessary for the proposed non-stationary massive MIMO channel model. There are two observations that should be noticed in Fig. 7. First, the PDFs of Doppler frequency at different time instants vary because of the non-stationary properties on the time axis. Namely, the WSS condition on the time domain is not available as a consequence of time-variant properties and the inclusion of birth-death process on the time axis. The factor $e^{-\lambda_{R}\left(\|\mathbf{v}\| \Delta t+P_{F}\left(\left\|\mathbf{v}_{n}^{T}\right\|+\left\|\mathbf{v}_{n}^{R}\right\|\right) \Delta t / D_{c}^{s}\right)}$ of the temporal ACF in (50) with respect to $\Delta t$ is equivalent to a translation in the Fourier transform domain. Moreover, simulation models align well with theoretical models as shown in Figs. 6 and 7.

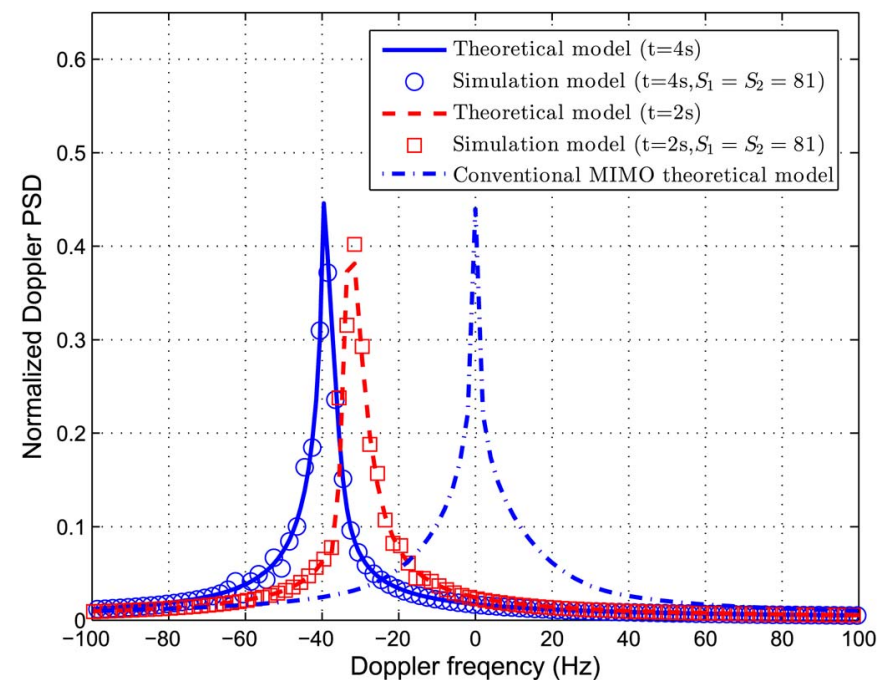

Fig. 7. The normalized Doppler PSD at different time instants $\left(M_{R}=32\right.$, $D=200 \mathrm{~m}, D_{c}^{a}=30 \mathrm{~m}, D_{c}^{s}=50 \mathrm{~m}, \varsigma=1 \mathrm{~s}, v_{A}^{T}=\pi / 3, v_{E}^{T}=\pi / 6, v_{A}^{R}=$ $\pi / 4, v_{E}^{R}=\pi / 4, \bar{\theta}_{1}^{T}=-1.03, \bar{\xi}_{1}^{T}=1.19, \bar{\theta}_{1}^{R}=1.65, \lambda=0.15 \mathrm{~m}, \lambda_{G}=$ $80 / \mathrm{m}, \lambda_{R}=4 / \mathrm{m},\left\|\mathbf{v}_{n}^{T}\right\|=\left\|\mathbf{v}_{n}^{R}\right\|=0.25 \mathrm{~m} / \mathrm{s}, P_{F}=0.3, f_{\max }=33.33 \mathrm{~Hz}$, $\alpha_{v}=\pi / 6, \kappa=5$, NLOS).

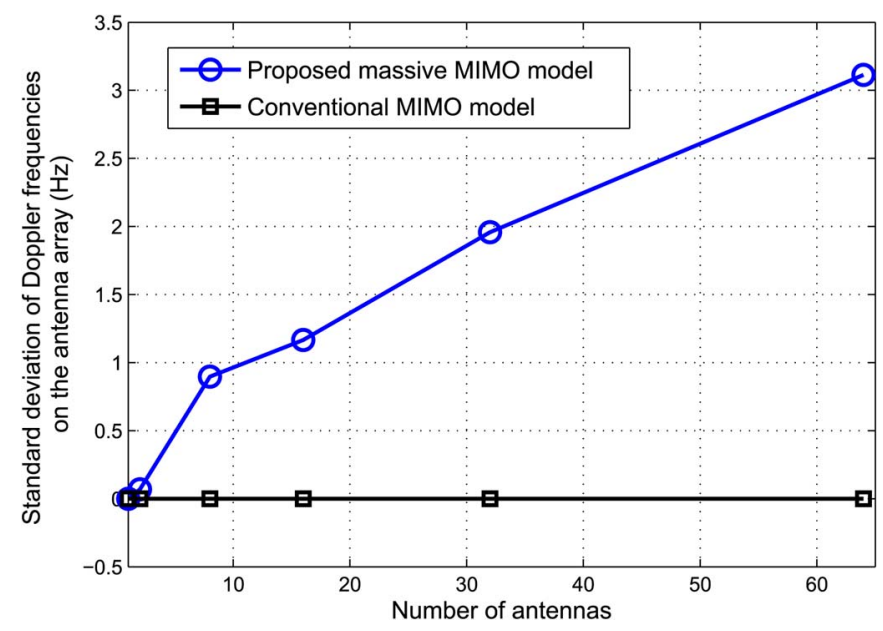

Fig. 8. Standard deviation of the Doppler frequencies on the antenna array $\left(D=200 \mathrm{~m}, D_{c}^{a}=30 \mathrm{~m}, D_{c}^{s}=50 \mathrm{~m}, \varsigma=1 \mathrm{~s}, v_{A}^{T}=\pi / 3, v_{E}^{T}=\pi / 6\right.$, $v_{A}^{R}=\pi / 4, v_{E}^{R}=\pi / 4, \bar{\theta}_{1}^{T}=-1.03, \bar{\xi}_{1}^{T}=1.19, \bar{\theta}_{1}^{R}=1.65, \bar{\xi}_{1}^{R}=0.16, \lambda=$ $0.15 \mathrm{~m},\left\|\mathbf{v}_{n}^{T}\right\|=\left\|\mathbf{v}_{n}^{R}\right\|=0.25 \mathrm{~m} / \mathrm{s}, P_{F}=0.3, f_{\max }=33.33 \mathrm{~Hz}, \alpha_{v}=$ $\pi / 6, \kappa=5$, NLOS).

In addition, Fig. 8 shows the standard deviation of Doppler frequencies on the antenna array. Conventional MIMO channel models assume far field condition which results in a constant Doppler frequency on the entire antenna array. Conversely, the near field condition is assumed in the proposed massive MIMO channel model. As a result, the Doppler frequencies for different antennas are different. Since the near field effect is more significant as the number of antennas grows, the standard deviation increases accordingly.

Furthermore, a comparison of condition numbers between the 2-D and 3-D models is shown in Fig. 9. Stronger correlations are observed in the 2-D model than the 3-D model due to the fact that clusters have higher probabilities to be correlated in a 2-D space than a 3-D space. However, this difference is 


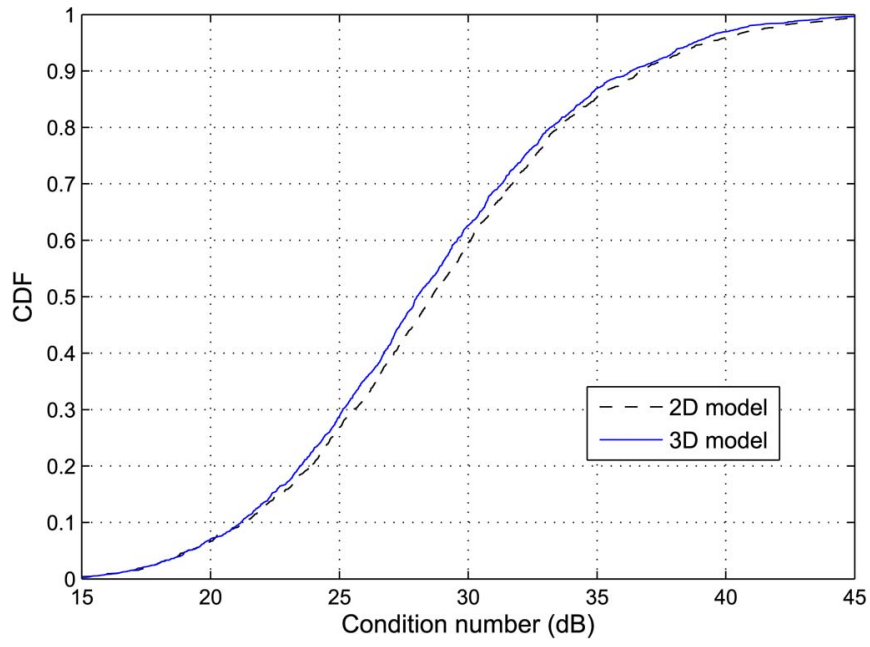

Fig. 9. Comparisons of CDFs of condition numbers between the 2-D and 3-D model $\left(M_{T}=4, M_{R}=32, D=200 \mathrm{~m}, D_{c}^{a}=30 \mathrm{~m}, D_{c}^{s}=50 \mathrm{~m}\right.$, $\varsigma=1 \mathrm{~s}, v_{A}^{T}=\pi / 3, v_{E}^{T}=\pi / 6, v_{A}^{R}=\pi / 4, v_{E}^{R}=\pi / 4, \bar{\theta}_{1}^{T}=-1.03, \bar{\xi}_{1}^{T}=$ $1.19, \bar{\theta}_{1}^{R}=1.65, t=4 \mathrm{~s}, \lambda=0.15 \mathrm{~m}, \lambda_{G}=80 / \mathrm{m}, \lambda_{R}=4 / \mathrm{m},\left\|\mathbf{v}_{n}^{T}\right\|=$ $\left.\left\|\mathbf{v}_{n}^{R}\right\|=0.25 \mathrm{~m} / \mathrm{s}, P_{F}=0.3, f_{\max }=33.33 \mathrm{~Hz}, \alpha_{v}=\pi / 6, \kappa=5, \mathrm{NLOS}\right)$.

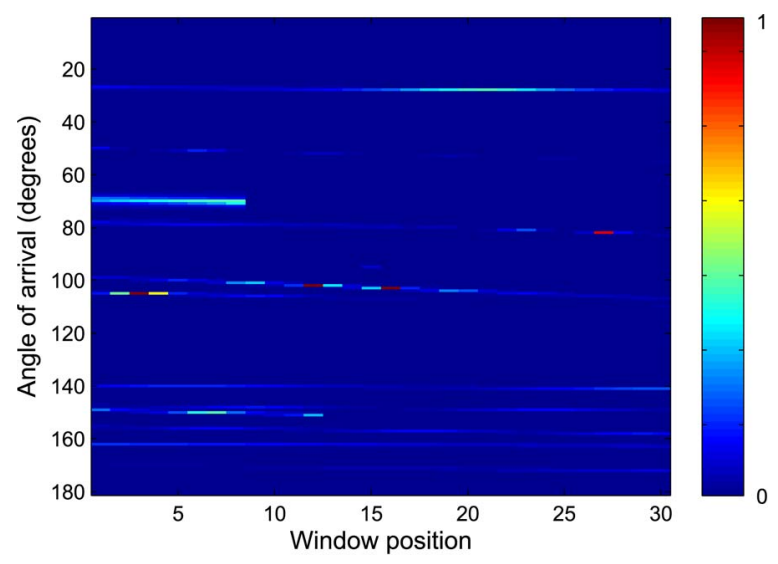

Fig. 10. A snap shot of the angular power spectrum of the receiver antenna array $\left(M_{T}=1, M_{R}=32, D=200 \mathrm{~m}, D_{c}^{a}=30 \mathrm{~m}, D_{c}^{s}=50 \mathrm{~m}, \varsigma=1 \mathrm{~s}, v_{A}^{T}=\right.$ $\pi / 3, v_{E}^{T}=\pi / 6, v_{A}^{R}=\pi / 4, v_{E}^{R}=\pi / 4, \bar{\theta}_{1}^{T}=-1.03, \bar{\xi}_{1}^{T}=1.19, \bar{\theta}_{1}^{R}=$ $1.65, t=4 \mathrm{~s}, \lambda=0.15 \mathrm{~m}, \lambda_{G} \stackrel{E}{=} 80 / \mathrm{m}, \lambda_{R}=4 / \mathrm{m}, f_{\max }=0 \mathrm{~Hz}$, NLOS).

relatively less significant because the random distribution of cluster locations partially averages out the impact.

Fig. 10 illustrates the non-stationary properties on the array axis in the form of the receiver angle power spectrum (APS). It should be noticed that the estimated angle here means the angle between the cluster and the receive antenna array. Here, the multiple signal classification (MUSIC) algorithm [42] is applied to AoA estimation. A sliding window formed by 3 consecutive receive antennas is shifted by 1 antenna at a time from the first to the last antenna. Consequently, for a 32-element antenna array, there are in total 30 window positions as Fig. 10 shows. Clusters appear and disappear on the array axis, which results in that different antennas may observe different sets of clusters. Additionally, angles of a number of clusters shift on the array axis due to the near field effect. Finally, receive power variations can be observed on the antenna array. Similar conclusions on these mentioned features of the proposed model were also observed in measurements in massive MIMO channels in [24].

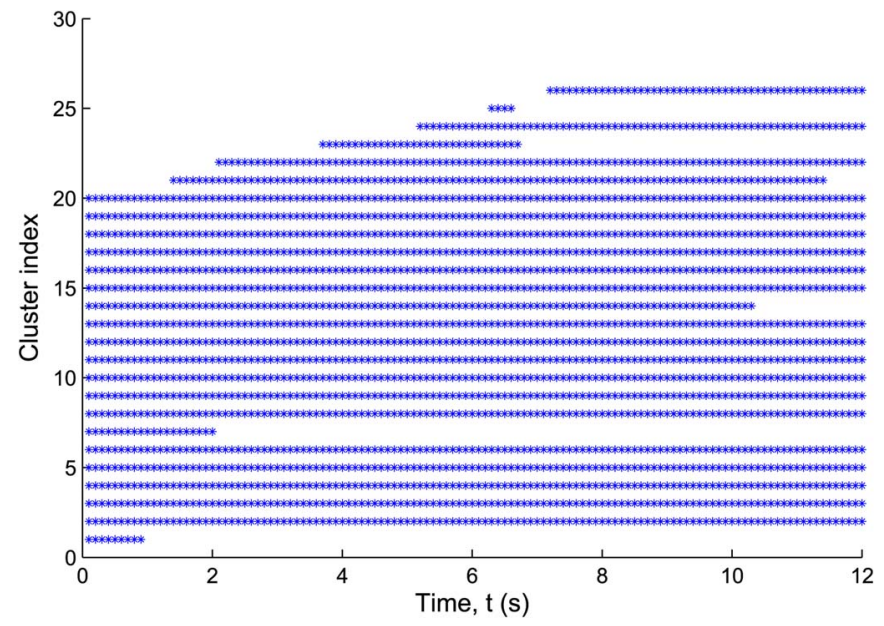

Fig. 11. Cluster evolution on the time axis $\left(D_{c}^{s}=50 \mathrm{~m}, \varsigma=1 \mathrm{~s}, \lambda=\right.$ $0.15 \mathrm{~m}, \lambda_{G}=80 / \mathrm{m}, \lambda_{R}=4 / \mathrm{m},\left\|\mathbf{v}_{n}^{T}\right\|=\left\|\mathbf{v}_{n}^{R}\right\|=0.25 \mathrm{~m} / \mathrm{s}, P_{F}=0.3$, $f_{\max }=33.33 \mathrm{~Hz}, \mathrm{NLOS}$ ).

Regarding cluster evolution on the time axis, an example of cluster sets in different time instants is shown in Fig. 11. Clusters evolve according to the birth-death process. Thus, it can be seen that there are clusters disappearing and new clusters appearing. In this case, the transmit and receive antenna arrays observe a time-variant set of clusters.

It is important to note that in the numerical analysis, parameters such as mean and standard deviation of azimuth AoAs/AoDs of the transmitter and receiver, mean and standard deviation of delays, maximum delay, spatial correlation distance, and cluster powers were generated according to the WINNER II channel model in [18]. The generation and recombination rates of clusters and percentage of moving clusters were adapted from [29] and [30]. However, certain parameters such as cluster distances to the transmitter or receiver were given based on reasonable assumptions, since we have not found any relevant measurement data so far. These parameters of the model can be further validated by measurements whenever available in the future.

\section{CONCLUSION}

Key characteristics of massive MIMO channels have not been captured by conventional MIMO channel models. In this paper, we have proposed a novel theoretical non-stationary 3-D wideband twin-cluster channel model along with the corresponding simulation model for massive MIMO systems with carrier frequencies in the order of GHz. Spherical wavefronts have been assumed to characterize near field effects resulting in AoA shifts, received power variations, and Doppler frequency variations on the antenna array. The impact of elevation angles of clusters on the correlation properties of the massive MIMO channel model has been studied. Most importantly, nonstationary properties on both the time and array axes have been modeled by birth-death processes. The proposed massive MIMO channel model is able to describe not only the appearance and disappearance of clusters on the time axis, but also the cluster evolution on the array axis, which is normally not included in conventional MIMO channels. Moreover, it has 
been shown that the channel characteristics of the simulation model are consistent with those of the theoretical model. In addition, important channel features of massive MIMO channels are characterized by the proposed models, which may serve as a design framework to model massive MIMO channels. Finally, certain parameters of the proposed channel model need to be further validated by relevant channel measurements, which will be our future work when such channel measurements become available in the literature.

\section{REFERENCES}

[1] G. J. Foschini and M. J. Gans, "On limits of wireless communications in a fading environment when using multiple antennas," Wireless Pers. Commun., vol. 6, no. 3, pp. 311-335, Mar. 1998.

[2] D. Gesbert, M. Shafi, D. Shiu, P. J. Smith, and A. Naguib, "From theory to practice: An overview of MIMO space-time coded wireless systems," IEEE J. Sel. Areas Commun., vol. 21, no. 3, pp. 281-302, Apr. 2003.

[3] G. J. Foschini, "Layered space-time architecture for wireless communication in a fading environment when using multielement antennas," Bell Labs Tech. J., vol. 1, no. 2, pp. 41-59, 1996.

[4] E. G. Larsson, F. Tufvesson, O. Edfors, and T. L. Marzetta, "Massive MIMO for next generation wireless systems," IEEE Commun. Mag., vol. 52, no. 2, pp. 186-195, Feb. 2014.

[5] F. Rusek et al., "Scaling up MIMO: Opportunities and challenges with very large arrays," IEEE Signal Process. Mag., vol. 30, no. 1, pp. 40-60, Jan. 2012.

[6] J. Hoydis, K. Hosseini, S. ten Brink, and M. Debbah, "Making smart use of excess antennas: Massive MIMO, small cells, TDD," Bell Labs Tech. J., vol. 18, no. 2, pp. 5-21, Sep. 2013.

[7] Nokia Solution Networks, 2020: Beyond 4G Radio Evolution for the Gigabit Experience. [Online]. Available: http://nsn.com/zh/file/15036/ 2020-beyond-4g-radio-evolution-for-the-gigabit-experience

[8] C.-X. Wang et al., "Cellular architecture and key technologies for 5G wireless communication networks," IEEE Commun. Mag., vol. 52, no. 2, pp. 122-130, Feb. 2014.

[9] C.-X. Wang, X. Hong, H. Wu, and W. Xu, "Spatial temporal correlation properties of the 3GPP spatial channel model and the Kronecker MIMO channel model," EURASIP J. Wireless Commun. Netw., vol. 2007, no. 1, pp. 39871-1-39871-9, Feb. 2007.

[10] M. Patzold, Mobile Radio Channels, 2nd ed. West Sussex, U.K.: Wiley, 2012.

[11] X. Cheng, C.-X. Wang, D. I. Laurenson, S. Salous, and A. v. vasilakos, "An adaptive geometry-based stochastic model for non-isotropic MIMO mobile-to-mobile channels," IEEE Trans. Wireless Commun., vol. 8, no. 9, pp. 4824-4835, Sep. 2009.

[12] C.-X. Wang, X. Cheng, and D. I. Laurenson, "vehicle-to-vehicle channel modeling and measurements: Recent advances and future challenge," IEEE Commun. Mag., vol. 47, no. 11, pp. 96-103, Nov. 2009.

[13] C.-X. Wang et al., "Cooperative MIMO channel models: A survey," IEEE Commun. Mag., vol. 48, no. 2, pp. 80-87, Feb. 2010.

[14] X. Cheng et al., "Cooperative MIMO channel modeling and multi-link spatial correlation properties," IEEE J. Sel. Areas Commun., vol. 30, no. 2, pp. 388-396, Feb. 2012

[15] X. Cheng et al., "Wideband channel modeling and ICI cancellation for vehicle-to-vehicle communication systems," IEEE J. Sel. Areas Commun., vol. 31, no. 9, pp. 434-448, Sep. 2013.

[16] X. Cheng, C.-X. Wang, B. Ai, and H. Aggoune, "Envelope level crossing rate and average fade duration of non-isotropic vehicle-to-vehicle Ricean fading channels," IEEE Trans. Intell. Transp. Syst., vol. 15, no. 1, pp. 6272, Feb. 2014

[17] Spatial Channel Model for Multiple Input Multiple Output (MIMO) Simulations, 3GPP T.S. 25.996 v11.0.0, 2012.

[18] P. Kyosti et al., WINNER II Channel Models, Sep. 2007, WINNER D1.1.2 ver 1.1. [Online]. Available: http://www.ist-winner.org/ WINNER2-Deliverables/D1.1.2v1.1.pdf

[19] "Guidelines for evaluation of radio interface technologies for IMTadvanced," Geneva, USA, Rep. ITU-R M.2135-1, 2009.

[20] A. Paulraj, R. Nabar, and D. Gore, Introduction to Space-Time Wireless Communications. Cambridge, U.K.: Cambridge Univ. Press, 2008.

[21] C. K. Wen, S. Jin, and K. K. Wong, "On the sum-rate of multiuser MIMO uplink channels with jointly-correlated Rician fading," IEEE Trans. Commun., vol. 59, no. 10, pp. 2883-2895, Oct. 2011.
[22] X. Hong, C.-X. Wang, B. Allen, and W. Malik, "A correlation based double-directional stochastic channel model for multiple-antenna UWB systems," IET Microw., Antennas Propag., vol. 1, no. 6, pp. 1182-1191, Dec. 2007.

[23] D. Tse and P. viswanath, Fundamentals of Wireless Communication. Cambridge, U.K.: Cambridge Univ. Press, 2005.

[24] S. Payami and F. Tufvesson, "Channel measurements and analysis for very large array systems at $2.6 \mathrm{GHz}$," in Proc. 6th Eur. Conf. Antennas Propag., Prague, Czech Republic, Mar. 2012, pp. 433-437.

[25] X. Gao, F. Tufvesson, O. Edfors, and F. Rusek, "Measured propagation characteristics for very-large MIMO at $2.6 \mathrm{GHz}$," in Conf. Rec. 46th Annu. Asilomar Conf. Signals, Syst., Comput., Pacific Grove, CA, USA, Nov. 2012, pp. 295-299.

[26] A. D. Yaghjian, "An overview of near-field antenna measurements," IEEE Trans. Antennas Propag., vol. 34, no. 1, pp. 30-45, Jan. 1986.

[27] J.-S. Jiang and M. A. Ingram, "Spherical-wave model for short-range MIMO," IEEE Trans. Commun., vol. 53, no. 9, pp. 1534-1541, Sep. 2005.

[28] F. Bohagen, P. Orten, and G. E. Oien, "Modeling of line-of-sight $2 \times 2$ MIMO channels spherical versus plane waves.," in Proc. IEEE PIMRC, Helsiniki, Finland, Sep. 2006, pp. 1-5.

[29] T. Zwick, C. Fischer, D. Didascalou, and W. Wiesbeck, "A stochastic spatial channel model based on wave-propagation modeling," IEEE J. Sel. Areas Commun., vol. 18, no. 1, pp. 6-15, Jan. 2000.

[30] T. Zwick, C. Fischer, and W. Wiesbeck, "A stochastic multipath channel model including path directions for indoor environments," IEEE J. Sel. Areas Commun., vol. 20, no. 6, pp. 1178-1192, Aug. 2002.

[31] C. C. Chong, C. M. Tan, D. I. Laurenson, S. McLaugnlin, and M. A. Beach, "A novel wideband dynamic directional indoor channel model based on a Markov process," IEEE Trans. Wireless Commun., vol. 4, no. 4, pp. 1539-1552, Jul. 2005.

[32] F. Babich and G. Lombardi, "A Markov model for the mobile propagation channel," IEEE Trans. Veh. Technol., vol. 48, no. 1, pp. 63-73, Jan. 2000.

[33] A. Kuchar, J.-P. Rossi, and E. Bonek, "Directional macro-cell channel characterization from urban measurements," IEEE Trans. Antennas Propag., vol. 48, no. 2, pp. 137-146, Feb. 2000.

[34] H. Hofstertter, A. F. Molisch, and N. Czink, "A twin-cluster MIMO channel model," in Proc. EuCAP, Nice, France, Nov. 2006, pp. 1-8.

[35] L. Liu et al., "The COST 2100 MIMO channel model," IEEE Commun. Mag., vol. 19, no. 6, pp. 92-99, Dec. 2012.

[36] R. verdone and A. Zanella, Pervasive Mobile and Ambient Wireless Communications: COST Action 2100. London, U.K.: Springer-Verlag, 2012.

[37] T. A. Thomas et al. " 3D Extension of the 3GPP/ITU channel model," in Proc. IEEE VTC-Spring, Dresden, Germany, Jun. 2013, pp. 1-5.

[38] K. Kalliola et al., "3-D double-directional radio channel characterization for urban macrocellular applications," IEEE Trans. Antennas Propag., vol. 51, no. 11, pp. 3122-3133, Nov. 2003.

[39] Y. Yuan, C.-X. Wang, X. Cheng, B. Ai, and D. I. Laurenson, "Novel 3D geometry-based stochastic models for non-isotropic MIMO vehicle-tovehicle channels," IEEE Trans. Wireless Commun., vol. 14, no. 1, pp. 298 309, Jan. 2014

[40] A. Papoulis and S. U. Pillai, Probability, Random variables, Stochastic Processes, 4th ed. New York, NY, USA: McGraw-Hill, 2002.

[41] A. Abdi and M. Kaveh, "A space-time correlation model for multielement antenna systems in mobile fading channels," IEEE J. Sel. Areas Commun., vol. 20, no. 3, pp. 550-560, Apr. 2002.

[42] A. F. Molisch, Wireless Communications, 2nd ed. West Sussex, U.K. Wiley, 2005

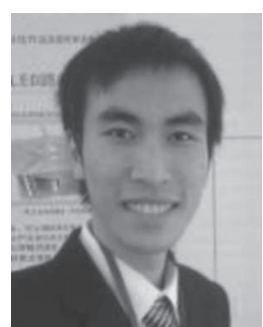

Shangbin $\mathrm{Wu}$ received the B.S. degree in communication engineering from South China Normal University, Guangzhou, China, in 2009 and the M.Sc. degree in wireless communications (with distinction) from the University of Southampton, Southampton, U.K., in 2010 . He is currently working toward the Ph.D. degree at Heriot-Watt University, Edinburgh, U.K.

From 2010 to 2011, he worked as an LTE R\&D Engineer responsible for LTE standardization and system-level simulation in New Postcom Equipment Ltd., Guangzhou. From October 2011 to August 2012, he was with Nokia Siemens Network, where he worked as an LTE Algorithm Specialist, mainly focusing on LTE radio resource management algorithm design and systemlevel simulations. His research interests include wireless channel modeling and simulation, spatial modulation, and massive multiple-input multiple-output systems. 


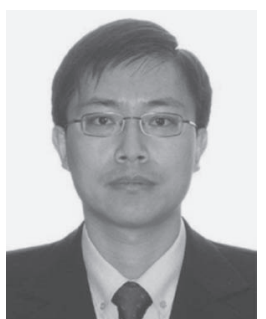

Cheng-Xiang Wang (S'01-M'05-SM'08) received the B.Sc. and M.Eng. degrees in communication and information systems from Shandong University, Jinan, China, in 1997 and 2000, respectively, and the Ph.D. degree in wireless communications from Aalborg University, Aalborg, Denmark, in 2004.

Since 2005, he has been with Heriot-Watt University, Edinburgh, U.K., where he was also promoted as a Professor in 2011. He is also an Honorary Fellow of the University of Edinburgh, Edinburgh, and a Chair/Guest Professor with Shandong University, and with Southeast University, Nanjing, China. From 2001 to 2005, he was a Research Fellow with the University of Agder, Grimstad, Norway. In 2004, he was a Visiting Researcher with Siemens AG-Mobile Phones, Munich, Germany. From 2000 to 2001, he was a Research Assistant with the Hamburg University of Technology, Hamburg, Germany. He is the Editor of one book. He has published one book chapter and over 200 papers in refereed journals and conference proceedings. His research interests include wireless channel modeling and simulation, green communications, cognitive radio networks, vehicular communication networks, massive multiple-input multiple-output systems, and fifth-generation wireless communications.

Prof. Wang is a Fellow of the Institution of Engineering and Technology and the HEA and a member of the Engineering and Physical Research Council Peer Review College. He has served as an Editor for eight international journals, including the IEEE TRANSACTIONS ON VEHICULAR TECHNOLOGY (since 2011) and the IEEE TRANSACTIONS ON WIRELESS COMMUNICATIONS (2007-2009). He was the lead Guest Editor for the IEEE JOURNAL ON SELECTED AREAS IN COMMUniCATIONS, Special Issue on vehicular Communications and Networks. He has served as a Technical Program Committee (TPC) Member, a TPC Chair, and a General Chair for more than 70 international conferences. He received the Best Paper Awards from IEEE Globecom 2010, IEEE ICCT 2011, ITST 2012, and IEEE VTC 2013-Spring.

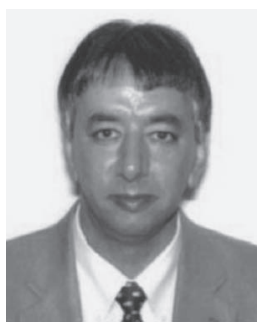

el-Hadi M. Aggoune (M'00-SM'09) received the $\mathrm{Ph} . \mathrm{D}$. degree in electrical engineering from the University of Washington (UW), Seattle, WA, USA. He is a Professional Engineer registered in the State of Washington and a winner of the IEEE Professor of the Year Award, UW Branch. He is listed as Inventor in a patent assigned to Boeing. He is currently a Professor and a Director of the Sensor Networks and Cellular Systems Research Center, University of Tabuk, Tabuk, Saudi Arabia. His research interests include modeling and simulation of large-scale networks, sensors, visualization, and control and energy.

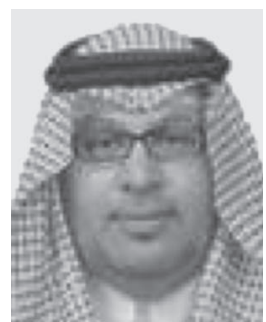

Mohammed M. Alwakeel (SM'14) was born in Tabuk, Saudi Arabia, in 1970. He received the B.S degree in computer engineering and the M.S. degree in electrical engineering from King Saud University, Riyadh, Saudi Arabia, in 1993 and 1998, respectively, and the $\mathrm{Ph} . \mathrm{D}$. degree in electrical engineering from Florida Atlantic University, Boca Raton, FL, USA, in 2005. From 1994 to 1998, he was a Communications Network Manager at The National Information Center, Saudi Arabia. From 1999 to 2001, he was with King Abdulaziz University, Jeddah, Saudi Arabia. From 2009 to 2010, he was an Associate Professor and the Dean of the College of Computers and Information Technology, University of Tabuk. He is currently the Vice Rector for Development and Quality of the University of Tabuk. His current research interests include teletraffic analysis, mobile satellite communications, sensor networks, and cellular systems.

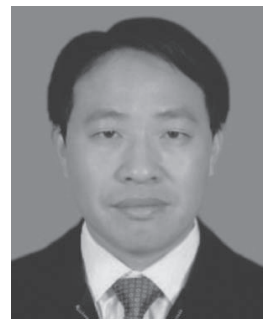

Yejun He (SM'09) received the B.S. degree from Huazhong University of Science and Technology, Wuhan, China, in 1994; the M.S. degree in communication and information systems from Wuhan University of Technology, Hubei, China, in 2002; and the Ph.D. degree in information and communication engineering from Huazhong University of Science and Technology, Hubei, China, in 2005.

From September 2005 to March 2006, he was a Research Associate with the Department of Electronic and Information Engineering, the Hong Kong Polytechnic University, Kowloon, Hong Kong. From April 2006 to March 2007, he was a Research Associate with the Department of Electronic Engineering, Faculty of Engineering, The Chinese University of Hong Kong, Shatin, Hong Kong. Since 2011, he has been a Professor of information and communication engineering with Shenzhen University, Shenzhen, China. From July 2012 to August 2012, he was a Visiting Professor with the Department of Electrical and Computer Engineering, University of Waterloo, Waterloo, ON, Canada. From October 2013 to October 2014, he was an Advanced Visiting Scholar (a Visiting Professor) with the School of Electrical and Computer Engineering, Georgia Institute of Technology, Atlanta, GA, USA. His research interests include channel coding and modulation, MIMO-OFDM wireless communications, space-time processing, smart antennas, etc. He is serving/served as a Reviewer/Technical Program Committee member/Session Chair for various journals and conferences, including the IEEE TRANSACTIONS ON VEHICULAR TECHNOLOGY, IEEE TRANSACTIONS ON COMMUNICATIONS, IEEE COMMUNICATIONS LETTERS, International Journal of Communication Systems, Wireless Communications and Mobile Computing, Wireless Personal Communication, KSII Transactions on Internet and Information Systems, IEEE VTC, IEEE PIMRC, IEEE WCNC, IEEE WCSP, etc. He is the Principal Investigator of more than ten current or finished research projects. He has authored or coauthored more than 70 research papers and applied for 12 patents since 2002 . He has been an Associate Editor of Security and Communication Networks since 2012. 